\title{
Plasmonic Metallic Heteromeric Nanostructures
}

\author{
Guangchao Zheng, ${ }^{a *}$ Stefanos Mourdikoudis ${ }^{b, c}$, Zhicheng Zhang ${ }^{d *}$
}

Dr. G. Zheng

aSchool of Physics and Microelectronics, Zhengzhou University, Zhengzhou, 450001 China

Email: zhengguangchao2008@gmail.com

Dr. S. Mourdikoudis

bBiophysics Group, Department of Physics and Astronomy, University College London (UCL), London, United Kingdom

${ }^{c}$ UCL Healthcare Biomagnetic and Nanomaterials Laboratories, London, United Kingdom

Prof. Z. Zhang

dTianjin Key Laboratory of Molecular Optoelectronic Sciences, Department of Chemistry, School of Science, Tianjin University \& Collaborative Innovation Center of Chemical

Science and Engineering, Tianjin 300072, China

Email: zczhang19@tju.edu.cn

Keywords: plasmonic heteromers, seed-mediated growth, site-selective growth, interface, heterostructure

Binary, ternary and other high-order plasmonic heteromers possess remarkable physical and chemical properties, enabling them to be used in numerous applications. Seed-mediated approach is one of the most promising and versatile routes to produce plasmonic heteromers. Selective growth of one or multiple domains on desired sites of noble metal, semiconductor or magnetic seeds would form desired heteromeric nanostructures with multiple functionalities and synergistic effects. In this review, the challenges for the synthetic approaches are discussed in respect to the tuning the thermodynamics, as well as the kinetic properties (e.g., $\mathrm{pH}$, temperature and injection rate, among others). Then, plasmonic heteromers with their structure advantages displaying unique and excellent activities compared to other hybrid nanostructures (e.g., core-shell, alloy) has been strengthened. Some of the main most recent applications of plasmonic heteromers are also presented. Finally, perspectives for further exploitation of plasmonic heteromers are demonstrated. The goal of this review is to provide the main current know-how on the synthesis routes of plasmonic heteromers in a summarized manner, with the aim of tailoring their performances and extending their applications. 


\section{WILEY-VCH}

\section{Introduction}

Metal nanomaterials exhibit unique physical and chemical properties. For example, bulk gold is inert, but it exhibits excellent catalytic properties at the nanoscale or single atom level. ${ }^{[1]}$ Compared with monometallic nanocrystals, hybrid nanoparticles (NPs), composed of a wide range of different metal elements, not only simply possess all the functions of their different nanodomains, but also exhibit new or enhanced performances and functionalities. Hybrid NPs are produced in the form of core-shell, ${ }^{[2]}$ alloy, ${ }^{[3]}$ hollow NPs, nanoboxes, ${ }^{[4]}$ and heteromers. ${ }^{[5]}$ In contrast to other types of hybrid NPs, heteromers demonstrate an excellent structure effect, enhancing their catalytic, optical, and magnetic properties. Especially, metal inorganic heteromers composed of at least one plasmonic nanodomain, called plasmonic metallic heteromers, are particularly attractive materials thanks to their localized surface plasmon resonance (LSPR) properties. LSPR is resulted from the collective oscillation of conduction electrons on the surface of plasmonic nanocrystals, in response to the incident light at their resonance wavelength. It can be decayed by the radiated electromagnetic field or by the formation of hot charges. These can enhance or induce chemical reactions of the reactant molecules on the NPs surface. ${ }^{[6]}$ When plasmonic nanocrystals incorporated with other metals, LSPR will be decayed into the electromagnetic field or hot electrons, which dominated most nanophotonic applications. To discover the plasmonic nanocrystal based applications, it is necessary to summarize the synthetic approaches and application of plasmonic hybrid NPs. Especially, plasmonic heteronanostructures shows their unique structure effect on their properties, which is dictated in the next. Plasmonic heteromeric nanostructures include binary, ternary and other high-order plasmonic heteromers (Figure 1). Overall, various types of plasmonic heteromers have already been used in several applications, such as, plasmon-enhanced photocatalysis, ${ }^{[7]}$ sensing, ${ }^{[8]}$ imagingguided cancer therapy ${ }^{[9]}$, electrocatalysis,,${ }^{[10]}$ chiroptical activity, ${ }^{[11]}$ etc. 


\section{WILEY-VCH}

With the development of a large variety of synthetic approaches and characterization technologies, nanocrystals with well-defined size, morphology, and internal structure, have already been synthesized in a controlled way in a big extent. So far, many research groups around the world have focused on morphology and composition control of plasmonic heteromers, resulting in more complex structures, with

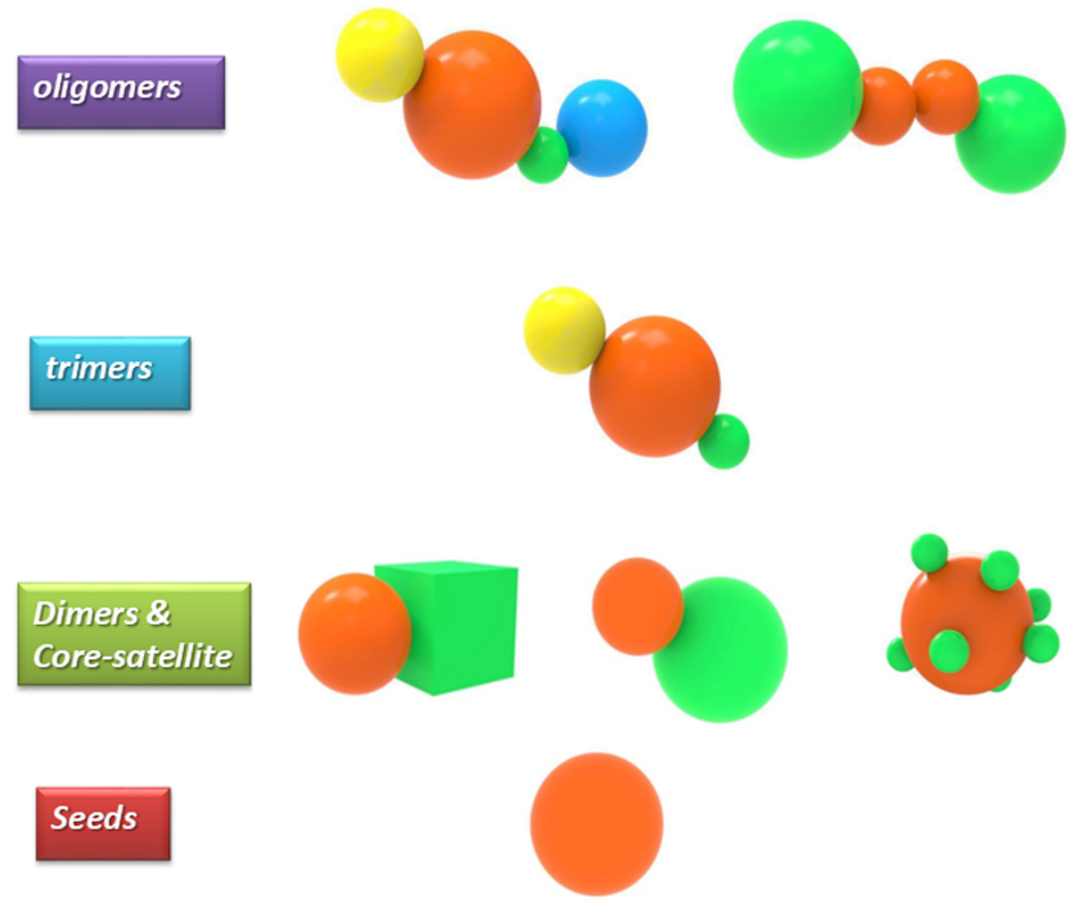

Figure 1: Schematic illustrations of dimers \& core-satellite, trimers, and oligomer plasmonic heteromeric nanostructures, with at least one plasmonic nanodomain.

improved functionalities. Until now, numerous synthetic approaches to acquire plasmonic metallic heteromers have been established, including template-directed growth, ${ }^{[12]}$ self-assembly, ${ }^{[13]}$ one-pot synthesis,,${ }^{[14]}$ microfluidics, ${ }^{[15]}$ and seed-mediated routes. In particular, seed-mediated approach was firstly proposed by Murphy and his colleagues for the production of Au nanorods, in 2001. ${ }^{[16]}$ The production of the initial seeds and the subsequent growth steps for the second element were separated in different space and time intervals. Since then, several groups further developed the seed-mediated growth method to successfully obtain gold prisms, stars, cubes, concave or convex NPs, as well as core-shell, nanoboxes, nanoframes, heteromers, and other peculiar 


\section{WILEY-VCH}

morphologies. ${ }^{[17]}$ With rational design of shape and application, seed-mediated approach has already been regarded as a highly efficient route to fabricate heteromeric nanostructures thanks to its time and space control. ${ }^{[3,5,18]}$ On one hand, the pre-formed nanocrystals, called "seeds", will provide primary sites for the heterogeneous nucleation and growth of secondary metallic adatoms because of their uniform and specific shapes. On the other hand, the thermodynamic and kinetic parameters can be finely tuned at the overgrowth stages to allow good control of growth modes resulting in the versatility of plasmonic heteromers. For example, the concentrations of seeds and added metal precursors can be quantitatively estimated to control the size and shape of secondary metallic nanodomains. So far, the morphologies of plasmonic heteromers have been accurately controlled via seed-mediated approaches.

It is well known that the properties of nanomaterials are strongly related to their compositions and morphologies. Especially, unique structure of plasmonic heteromers shows that they possess several phase segregated nanodomains via a solid-solid nanointerface, including metal-noble metal, metal-semiconductor, metal-magnetic component nanointerface. There are also plasmonic-polymer, plasmonic-silica and other heteromers which are not discussed in this review because of their different growth mechanisms compared to metal-metal nanointerface. ${ }^{[19]}$ The structure properties of plasmonic heteromers do not only include the LSPR feature, but also strengthen their arising chemical and physical properties compared to core-shell and alloy nanostructures. Under the laser irradiation, there are generated pairs of hot holes and hot electrons on the surface of plasmonic NPs. The generated hot electrons on plasmonic NPs surface can be transferred to secondary metal active sites, promoting the chemical reaction on the surface of the latter sites. Meanwhile, the left hot holes on the plasmonic NPs surface would be accumulated. In order to continuously supply the hot electrons, the left hot holes need to be neutralized by the electron donors. However, this refill process is prohibited by the completed shell of the plasmonic nanoparticle@metal core- 


\section{WILEY-VCH}

shell nanostructures. In contrast, the sacrificed hot electrons are directly refilled by the electron donors in the heteromeric nanostructures because these donors can directly contact with plasmonic NPs. Zheng et al. have found that the photocatalytic efficiency of Pt tip-on Au nanorods was improved compared to Au nanorods@Pt core-shell nanostructures. $^{[20]}$ In addition, localized near electromagnetic field of plasmonic nanodomains will enhance the optical absorption and the separation efficiency of hot electrons and hot holes of secondary metal nanodomains. Seh et al. have found that Au$\mathrm{TiO}_{2}$ heteromers exhibit higher efficiency in hydrogen evolution reaction compared to $\mathrm{Au}-\mathrm{TiO}_{2}$ core-shell nanostructures which is partly contributed to the larger dielectric mismatch between $\mathrm{Au}$ and $\mathrm{TiO}_{2}$ in the heteromers structures compared to that of coreshell structures. ${ }^{[21]}$ Secondly, the solid-solid interfaces between the different nanodomains of plasmonic heteromers can improve the electrocatalytic performance of the material in comparison to their individual counterparts. ${ }^{[22]}$ Tan et al. have reported that heterogeneous growth of $\mathrm{Pt}$ or $\mathrm{Pt}_{3} \mathrm{Ni}$ on the gold nanowires can overcome the dissolution and migration of $\mathrm{Pt}$, leading to the enhancement of catalytic efficiency and durability towards oxygen reduction reaction (ORR) because of the electron communication via interface and the higher potential range of Au. ${ }^{[23]}$ In another work, $\mathrm{Wu}$ et al. fabricated $\mathrm{Au}$ nanodomains grown on $\mathrm{Pt}_{3} \mathrm{Ni}$ nanoframes by the galvanic replacement reaction of $\mathrm{Au}$ precursors and $\mathrm{Ni}(0)$ displaying excellent selectivity, activity and durability in the hydrogenation of 4-nitrobenzaldehyde and the oxidation of methanol. The high selectivity is a result of the preferred absorbance of the nitro groups onto the Au nanodomains. Meanwhile, the enhanced activity is due to the electron communication between the $\mathrm{Au}$ nanodomains and $\mathrm{Pt}_{3} \mathrm{Ni}$ nanoframes and the suppression of the CO poisoning effect. ${ }^{[24]}$ Finally, the biological "water window" is located at the "800-1200 nm" region for the laser in biological tissues because of the strong fluorescence background and water interference. LSPR of plasmonic heteromers can be extended from the visible to near-infrared region, which can get fully use of the visible- 


\section{WILEY-VCH}

NIR light for the energy conversion from solar energy to chemical energy. ${ }^{[25]}$ However, the shell scattering in core-shell nanostructures would decrease the efficiency of LSPR or magnetic property. So, plasmonic domains are also one excellent candidate for multimode bioimaging.

Currently, there are many interesting research reports and several reviews which present critical issues of the syntheses, characteristics and applications of plasmonic heteromers. ${ }^{[26]}$ Especially, Xia and co-workers have made important contributions on ways to control seed-mediated synthetic routes in a qualitative and quantitative production manner. ${ }^{[27]}$ Although there are some interesting review papers discussing the synthesis and applications of hybrid NPs, ${ }^{[10 b, 26 b, 28]}$ in this review we focus on the type of plasmonic heteromeric nanostructures, avoiding overlaps with previous reports. Particularly we review the seed-mediated synthetic routes and uses of plasmonic metallic heteromers in a critical way. This piece of work aims to provide a better understanding of such modern category of nanomaterials.

\section{Seed-mediated approaches for synthesis of plasmonic metallic heteromeric} nanostructures: Thermodynamics vs Kinetics control

Seed-mediated approach is one of the most widely employed routes for the synthesis of plasmonic heteromers. ${ }^{[29]}$ The presence of seeds can decrease the activation energy of heterogeneous nucleation and growth as well as the critical concentration of secondary metal adatoms leading to the prohibition of the homogeneous nucleation and growth. The formed adatoms below supersaturation level produced by the photo- or chemicalreduction or thermal decomposition prefer heterogeneous growth rather than selfnucleation and growth on the seeds. ${ }^{[28 f]}$ A common way to use this synthesis mode is the so-called "one-pot" method. ${ }^{[30]}$ In this method, all reagents are added into the same reaction flask and the precursors of the first metal are reduced faster than the precursors of the second element, due to differences in their thermal decomposition easiness or reduction potentials. Thus, nucleation results in the formation of seeds of the first metal, 


\section{WILEY-VCH}

and the reduction of the precursor of the second metal will then lead to its heterogeneous nucleation on the preformed seeds. Growth of the second element on these seeds of the first metal will follow. A drawback of these one-pot strategies is the lack of precise control and rational design of the features of the seeds.

In this review, the seed-mediated approach involves a series of different stages, which are carried out in separate time intervals and sometimes using multi-pot rather than one-pot strategies. Seeds are produced with well-defined features, afterwards they are introduced into a growth solution containing adatoms, or, reversely adatoms are added into a growth solution which contains seeds. The overgrowth process can be well controlled by the thermodynamics and kinetics. In order to avoid homogeneous nucleation, adatoms are often manipulated to favour the heterogeneous nucleation (or deposition) and growth at specific sites with high surface free energies (tips, edges, and uncapped regions) driven by both thermodynamics and kinetics control, which are analysed below. Seed-mediated approach minimizes the total Gibbs free energy, the sum of surface free energy, internal defects, strain energies and crystal phase, that are parameters related to thermodynamics. ${ }^{23 \mathrm{~d}, 27}$ The thermodynamics-based process is favoured by controlling factors as capping agents, temperature, $\mathrm{pH}$ value etc. While the kinetic control (e.g., injection rate control of adatoms) strongly depends on the experimental details. For example, in the course of nanocrystal synthesis, surfactants (capping agents, such as hexadecyl trimethyl ammonium bromide (CTAB), hexadecyl trimethyl ammonium cloride (CTAC), polyvinylpyrrolidone (PVP), proteins, and other molecules) prefer to adsorb on specific facets of mono-nanocrystals. This can play two essential roles in the growth mode of heteromers, including (i) soft template and capping agent providing colloidal stability, (ii) and modification of the Gibbs free energy of whole reaction systems promotes the formation of plasmonic heteromers which is favoured by the kinetics. ${ }^{[31]}$ Xiang et al. have reported that CTAB strongly bonded on the $\{110\}$ facets of Ag leads to the selective deposition of Ag around the Au NR, thereby 


\section{WILEY-VCH}

resulting in formation of slice-like Au-Ag heteromers. ${ }^{[32]}$ In another report, Zhang et al. have chosen a dye with thiol groups as a selective capping agent which drives the Ag nanodomains overgrowth on the tips of Au nanostars because the dye is only localized at the tips, thus forming Au-Ag satellite nanostructures. ${ }^{[33]}$ Therefore, one of the most common ways to adjust the thermodynamics and kinetics of whole synthesis systems, so as to control the morphologies of the resultant plasmonic heteromers, is the insertion of surfactants in the reaction process.

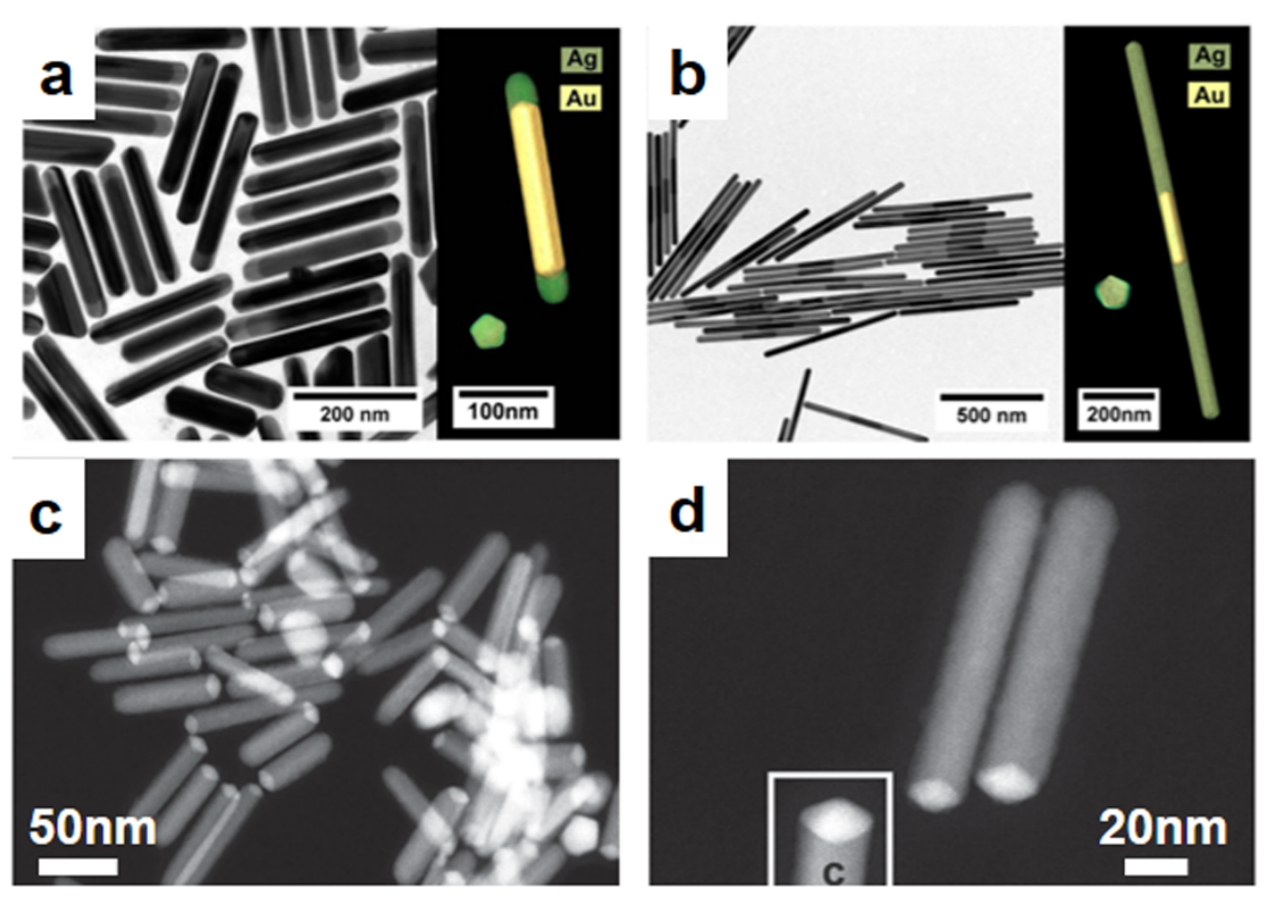

Figure 2 a) and b) TEM images of Au-Ag plasmonic heteromers. Reproduced with permission. ${ }^{[34]}$ a,b) Copyright 2015, American Chemical Society. c) and d) High-angle annular dark-field imaging (HAADF) (HADDF-STEM) images of $\mathrm{Pd}-\mathrm{Cu}$ plasmonic heteromers. Reproduced with permission. ${ }^{[35]} \mathrm{c}, \mathrm{d}$ ) Copyright 2016 , Wiley-VCH.

Especially, plasmonic heteromers are produced when the first metal seeds are fused with other phase segregated functional metal nanodomains at the desired sites via solidsolid nanointerface. Nanocrystals with narrow size monodispersity and homogeneous morphology constitute the primary foundation in the synthetic approaches. ${ }^{[36]}$ Seeds, used by the performed well-defined nanocrystals, usually possess desired facets or sites on which the adatoms selectively heterogeneous nucleate and grow. The construction of numerous kinds of plasmonic heteromers strongly depends on the quality of seeds (e.g., 


\section{WILEY-VCH}

twin planes, stacking faults and crystal phase). ${ }^{[37][38]}$ Liz-Marzan and co-workers have shown that penta-twinned Au nanorods used as seeds can induce a larger aspect ratio of $\mathrm{Au}-\mathrm{Ag}$ heteromers (Figure 2a,b). The formation of Au-Ag plasmonic heteromers is achieved due to the following reasons: (i) the capping agent, benzyldimethylhexadecylammonium chloride (BDAC), prefers to adsorb on the $\{100\}$ facets of penta-twinned Au nanorods, resulting in the polymeric growth mode of $\mathrm{Ag}$ rather than lateral growth. (ii) The growth rate is also governed by the slow addition of the precursors as well as the reducing agents and the temperature. The lowest growth rate promotes the polymeric growth mode and enhances the aspect ratio in which the penta-twinned nanorods promote the heterogeneous growth of the silver atoms on the tips of penta-twinned Au nanorods. ${ }^{[34]}$ Luo et al. have fabricated Pd-Cu heteromers by using Pd decahedral NPs as seeds in the presence of capping agent HDA, glucose, and $\mathrm{CuCl}_{2}$ (Figure 2c,d). Pd decahedral NPs provide active sites along $<110>$ direction due to the large lattice mismatch $(7.1 \%)$ between $\mathrm{Pd}$ and $\mathrm{Cu}$ and attachment of HAD on the $\{100\}$ facets. Besides, HDA can prevent the oxidation of surface atoms of $\mathrm{Cu}$, but it can be also used as capping agent attached on the $\{100\}$ facets and in this way it can favour the formation of $\mathrm{Pd}-\mathrm{Cu}$ penta-twinned heteromeric nanowires. ${ }^{[35]}$

The large mismatch between the seeds and secondary metallic nanodomains will cause great positive strain energy $\left(\gamma_{\text {strain }}\right)$ when the heterogeneous nucleation and growth of nanodomains are induced over the seeds surface. ${ }^{[39]}$ Low lattice mismatch and surface energy barrier usually facilitates adatoms homogeneous nucleation and conformal growth on the seeds surface to form hybrids. ${ }^{[28 a, 40]}$ Adatoms rotate themselves and interact with seeds step by step so as to decrease the lattice mismatch. However, there 


\section{WILEY-VCH}

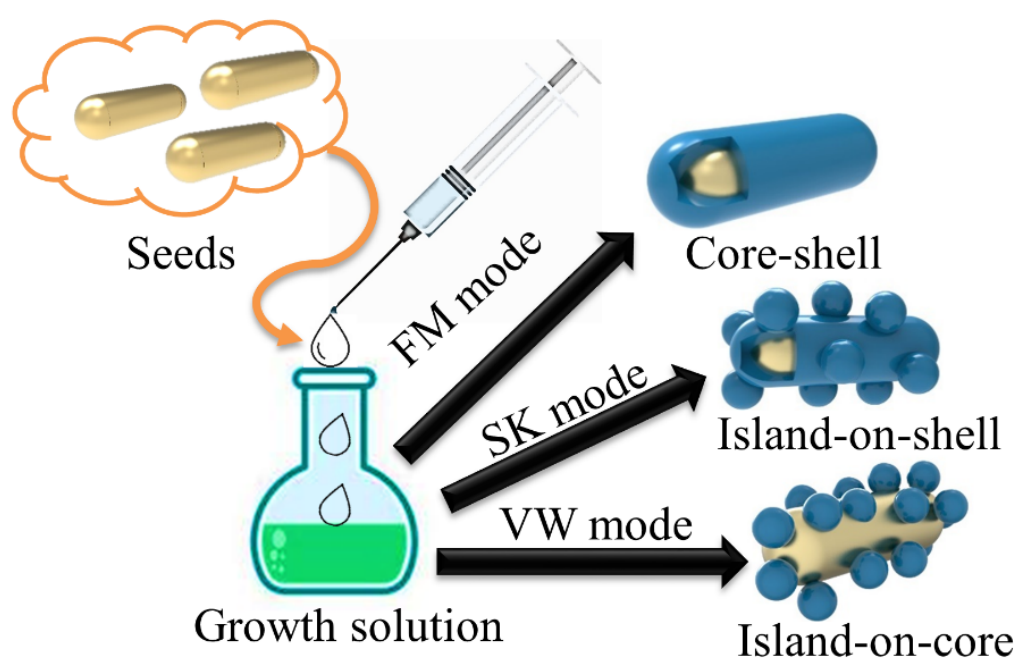

Figure 3. Schematic illustration of three different growth modes of plasmonic heteromers using seed-mediated approaches.

still is the effect of lattice mismatch. ${ }^{[41]}$ To continue relieving the interface strain, adatoms grow in an "island mode"-way on the surface of seeds. The new interfacial energy $\left(\gamma_{i}\right)$ resulting from the solid-solid nanointerface between adatoms and seeds should be taken into account in the total surface energy:

$$
\Delta \gamma=\gamma_{\text {adatoms }}+\gamma_{i}+\gamma_{\text {strain }}-\gamma_{\text {seeds }}
$$

As a result, different types of hybrid NPs, including core-shell (Frank-van der Merwe (FM) mode, $\Delta \gamma<<0$ ), island-on-shell (Stranski-Krastanov (SK) mode, $\Delta \gamma<0$ ), and island-on-core nanostructures (Volmer-Weber (VW) mode, $\Delta \gamma>0$ ) (Figure 3), are obtained by decreasing the Gibbs free energies, respectively. ${ }^{[42]}$ To achieve the formation of plasmonic heteromers, the total surface energy usually needs to be positive. Therefore, the thermodynamics and kinetics parameters should be designed to increase the surface energy of adatoms and interfacial energy in order to ensure values for $\Delta \gamma>0$. Morphology and composition controllability in plasmonic heteromers are also sensitive to kinetic control - the ratio of deposition rate and diffusion rate of adatoms which strongly depends on the experiments. Lim et al. have found that 


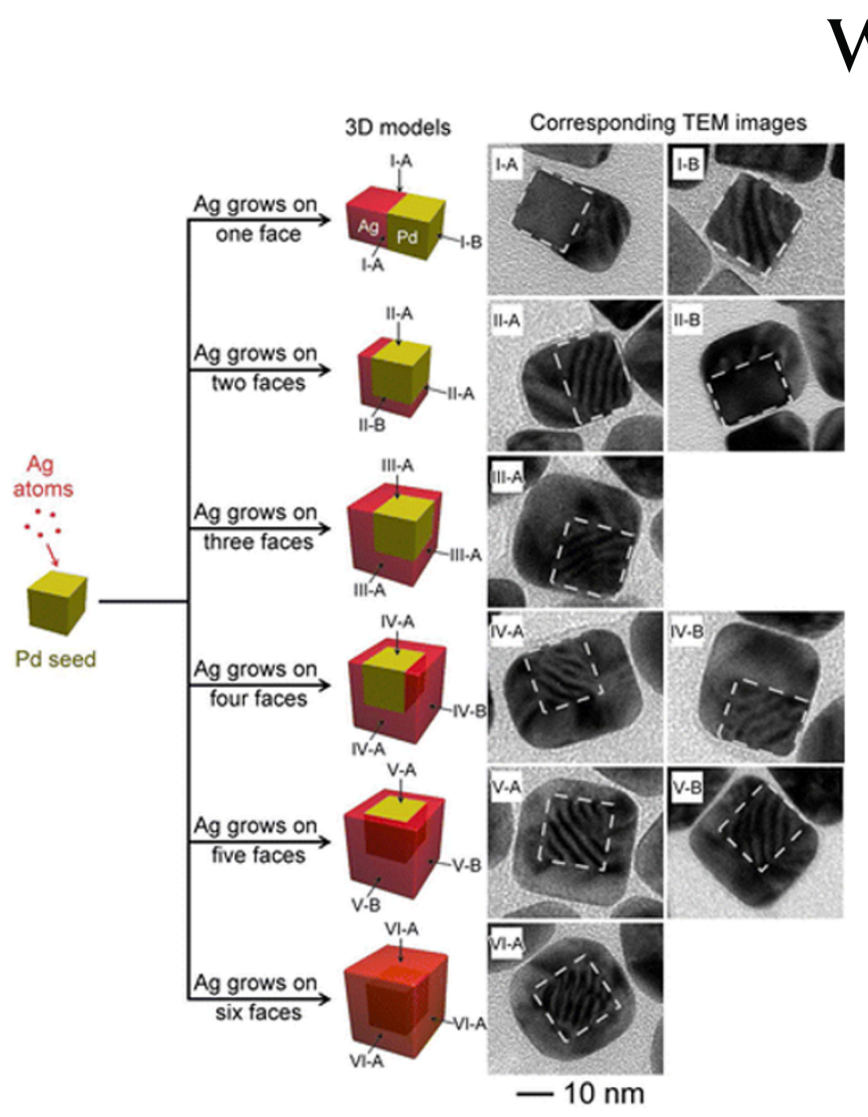

Figure 4. Schematic illustration showing the growth of Ag on selected facets of a cubic Pd seed. Reproduced with permission ${ }^{[43]}$. Copyright 2012, American Chemical Society.

low concentration of metal precursors can lead to the heterogenous growth of $\mathrm{Au}$ adatoms only onto one site of nanocubic Pd NPs in the presence of a weak reducing agent (citric acid), thus forming heterodimers. However, a relatively stronger reducing agent (ascorbic acid) favours the generation of core-shell nanostructures. ${ }^{[4]}$ An intriguing further endeavour is the selective growth of $\mathrm{Au}$ or $\mathrm{Ag}$ domains on $\mathrm{Pd}$ nanocubic seeds which is qualitatively and quantitatively characterized by Xia's group. ${ }^{[45]}$ The heterogrowth was controlled by the kinetic control (injection rate of the adatoms), favoured by the thermodynamics (reductant, capping agent, and reaction temperature) (Figure 4). ${ }^{[43]} \mathrm{Ag}$ would nucleate on one face, two faces or six faces of $\mathrm{Pd}$ NPs relying on the slow, moderate or fast injection rate of silver precursors, respectively. ${ }^{[46]}$ Furthermore, by selectively controlling the type and amount of capping agents/reductants, as well as the reaction temperature, Ag can nucleate and grow on any face of Pd NPs in order to multimeric nanostructures. As the concentration of PVP is 


\section{WILEY-VCH}

decreasing, a larger surface area of Pd nanocubic seeds becomes uncovered, thus providing new sites for the nucleation and growth of Ag domains. Besides, increasing the temperature would efficiently enhance the frequent collision between the Ag adatoms and Pd seeds thus facilitating the growth of Ag on more facets of Pd nanocubic seeds. ${ }^{[43]}$ All that can be explained based on the following proposed mechanism: once the $\mathrm{V}_{\text {deposition }} / \mathrm{V}_{\text {diffusion }}>>1$, surface diffusion could be ignored, and adatoms can only be deposited on the designed surface or corners. While $\mathrm{V}_{\text {deposition }} / \mathrm{V}_{\text {diffusion }}<<1$, on the contrary, the surface diffusion could dominate the growth and most of the adatoms can quickly migrate to the side facets and edges producing the core-shell NPs. On the conditions of the $\mathrm{V}_{\text {deposition }} / \mathrm{V}_{\text {diffusion }}$ slightly larger than 1 or lower than 1 , the resulting nanostructures could be concave or elongated with truncated nanostructures correspondingly. ${ }^{[45]}$

\subsection{Plasmonic-noble metal heteromers}

Due to the broad application in the field of catalysis, energy, sensing, and cancer therapy, noble metal nanoparticles (Pt, $\mathrm{Au}, \mathrm{Pd}, \mathrm{Ag}$ ) have been the focused subject of widespread studies. Plasmonic-noble metal heteromers can extend their applications by the merit of LSPR or solid-solid metal nanointerface.

Pt normally grows in "SK modes" or "VW mode" on Au seeds surface due to higher surface free energy of Pt. ${ }^{[6 a, 6 b, 14 a, 47]}$ Tian's group have shown that the overgrowth of Ag and Pd on the Au octahedral follows FW mode, while Pt prefers to grow with VW mode. Since the highest Pt-Pt bond energy results in its higher surface energy, which inhibits the diffusion rate of $\mathrm{Pt}$ adatoms on the seed surface. ${ }^{[48]}$ The synthetic approaches for the 


\section{WILEY-VCH}
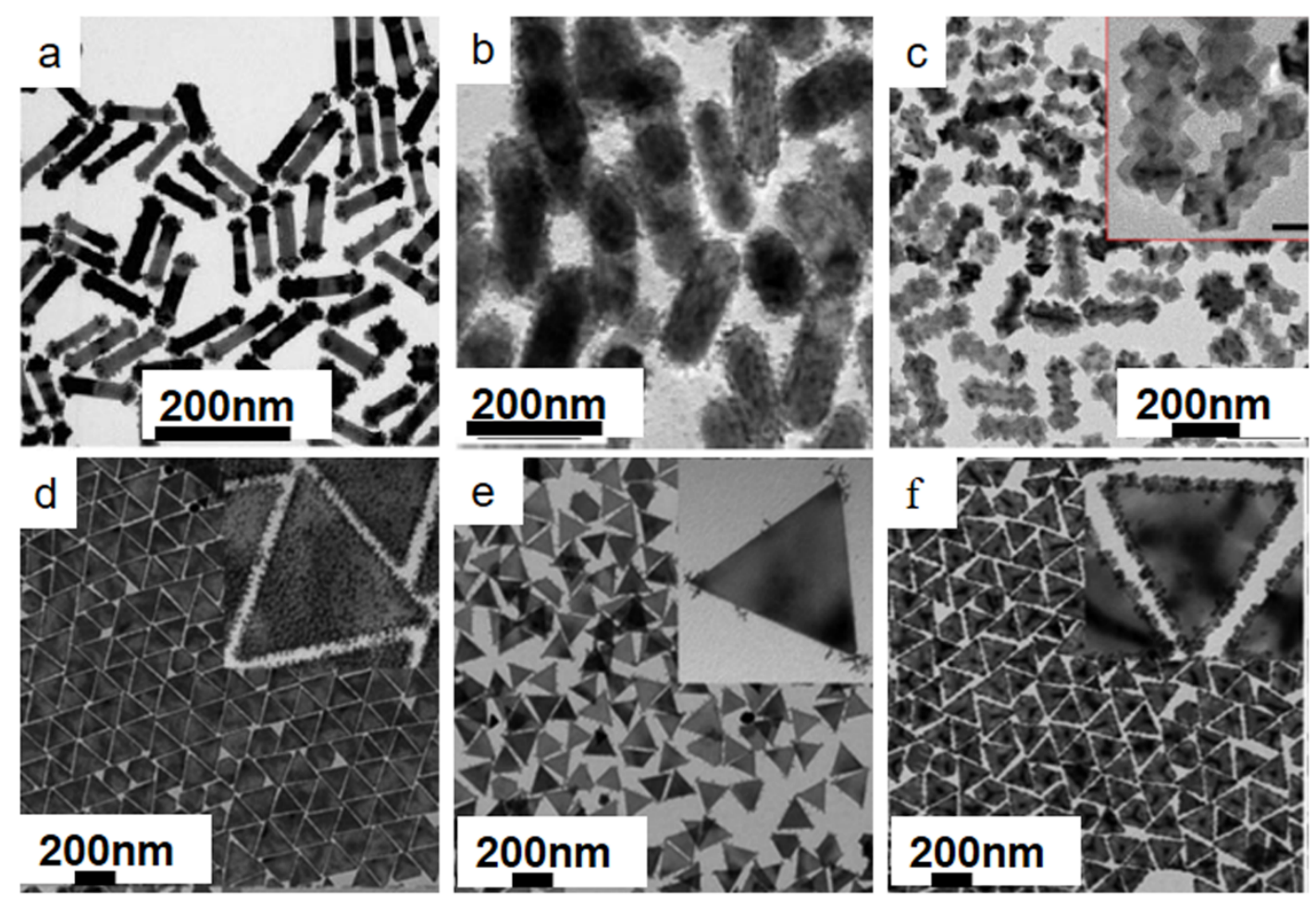

Figure 5. Synthesis of Au-Pt hybrid NPs. a) Pt tip-on Au nanorods. Reproduced with permission $^{[6 \mathrm{~b}]}$. Copyright 2007, Royal of Society of Chemistry. b) Pt nanodendrites covered-Au nanorods. Reproduced with permission ${ }^{[47 a]}$. Copyright 2014, Royal of Society of Chemistry. c) Pt covered-Au nanorods. Reproduced with permission ${ }^{[6 a]}$. Copyright 2014, American Chemical Society. d) Pt covered-Au nanoprisms; e) Pt tip-on $\mathrm{Au}$ nanoprisms; f) Pt edge-on $\mathrm{Au}$ nanoprisms. Reproduced with permission ${ }^{[47 \mathrm{e}]}$. d-f) Copyright 2016, American Chemical Society.

overgrowth of Pt adatoms on the plasmonic seeds with FM mode, VW mode, or SK mode can be controlled through adjusting the seeds surface energy by tuning the $\mathrm{pH}$ value and adding suitable ions and small molecules. A series of Au-Pt heteromers have been produced respectively. In the presence of $\mathrm{Ag}^{+}, \mathrm{Pt}$ adatoms prefer to deposit on $\{111\}$ and $\{100\}$ facets of Au nanorods, leading to nucleation and growth of Pt at the tips of gold nanorods resulting in dumbbell shape (Figure 5a). That is because the presence of $\mathrm{Ag}^{+}$hindered the deposition rate of $\mathrm{Pt}$ adatoms on side facets $\{110\} .{ }^{[49]} \mathrm{As}$ the ratio between the population of seeds and adatoms increases, the growth mode evolved from VW to SK modes. ${ }^{[6 b]}$ Increasing the number of Pt adatoms will lead to surface coverage of Pt nanodendrites (Figure 5b). Additionally, the one-dimensional growth mode of Au nanorods leads to tips of Au NRs which are usually occupied with 


\section{WILEY-VCH}

lower amount of capping agent than side facets. ${ }^{[50]}$ So, Pt adatoms initially prefer to nucleate and grow at the tips of Au nanorods. When the concentration of capping agent CTAB decreases to lower than critical micelle concentration $(c m c)$, CTAB on the sides cannot block the deposition of Pt adatoms leading to Pt-covered Au nanorods (Figure 5c). Counter ions, capping agent attaching on the higher-index facets of seeds have been well documented in many publications. ${ }^{[51]}$ A linear arrangement of Pt islands overgrows on the Au nanoprisms seeds because of the supramolecules formed by the capping agent. In contrast, Pt-covered $\mathrm{Au}$ nanoprisms were produced in the absence of iodide ions (Figure 5d). In Figure 5e, Pt tip-growth on Au nanoprisms was achieved in the presence of iodide ions. These ions initially adsorb on facets of Au nanoprisms forming AgI where they limit the nucleation and growth of Pt adatoms. ${ }^{[47 e]} \mathrm{Pt}$ edge-growth on Au triangular NPs are difficult to control in these conditions since the deposition rate of $\mathrm{Pt}$ is larger than the diffusion rate. Lou et al. have demonstrated the Pt edge-growth on Au triangular NPs when the capping agent CTAB is replaced by CTAC (Figure 5f). ${ }^{[47 e]}$ In that case, there is a large amount of count ions $\mathrm{Cl}^{-}$instead of $\mathrm{Br}^{-}$in the growth solution. $\mathrm{AgCl}$ is more difficult to be reduced because the value of $\mathrm{AgBr} / \mathrm{Ag}(0.071 \mathrm{~V}$ vs. SHE) is lower than $\mathrm{AgCl} / \mathrm{Ag}$ (0.222 V vs. SHE). Therefore, the diffusion rate of Pt adatoms increased leading to Pt 'moving' from the tips to the edges.

Low-coordination sites on the nanodendrites structure of seeds promote the heterogeneous nucleation and growth of Au adatoms. In one of our previous works, we have found that heterodimers and core-satellite hybrid NPs were obtained with different capping agents in the Au-Pt system. CTAB was used for the single-site growth mode while PVP was applied for multiple growth mode in the case of Pt-Au heterostructures (Figure 6a-f). CTAB was regarded as forming the complex of $\mathrm{CTA}^{+}-\mathrm{Au}$ which perplexed the tip-growth of the Au nanodomains due to the surface defects at the tips of the Pt nanodendrites. This resulted in the acquisition of Au-Pt nanodimers. In contrast, polyvinylpyrrolidone (PVP) resulted in a faster reaction rate when using a common gold 


\section{WILEY-VCH}

precursor, chloroauric acid, leading to the generation of $\mathrm{Pt} @ \mathrm{Au}$ core-satellite structures. $^{[52]}$ Tang and colleagues found that the pyridinium headgroup of cetylpyridinium chloride (CPC) can induce the self-organization of Pd nanoarrays on the surface of Au nanorods. Typical TEM characterization of Au-Pd plasmonic heteromers is shown in Figure $6 \mathrm{~g}$ and h. At $65{ }^{\circ} \mathrm{C}$, the formation of $\left[\mathrm{CPC}^{+}\right]_{2} \cdot\left[\mathrm{PdCl}_{4}{ }^{2-}\right]$ complexes in the micelles are sustainable for the diffusion and reduction of Pd precursors on $\mathrm{Au}$ nanorods seeds. Additionally, the head groups and counter ions of surfactants varies the topology and $c m c$ of surfactants, which influences the morphology of heterogeneous growth modes. ${ }^{[51 \mathrm{c}, 53]}$ Compared to the surfactants with long-chain alkyl groups (e.g., CTAC, CTAB), CPC with the polarizability and $\pi-\pi$ stacking interaction of aromatic headgroup, can make Pd randomly grow on the Au nanorod seeds. ${ }^{[54]}$ Furthermore, the counter ions $\left(\mathrm{Cl}^{-}\right)$of $\mathrm{CPC}$ bind more weakly on $\mathrm{Pd}$ surface compared to $\mathrm{Br}^{-}$of cetylpyridinium bromide (CPB). Therefore, more $\mathrm{Pd}$ adatoms are promoted to heterogeneously nucleate and grow on the Au seeds in the presence of CPC.

Most heteromers are produced under inert atmosphere, i.e. in the presence of Ar or $\mathrm{N}_{2}$ gases. ${ }^{[55]}$ Such conditions are necessary to prohibit the oxidation of nanoparticles at higher temperature. Zhu et al. have recently illustrated that the reaction atmosphere can affect the final morphology of hybrid NPs (Figure 7a-i). In the presence of reducing $\mathrm{H}_{2}$ atmosphere, the surface adsorbed $\mathrm{H}_{2}$ on FePd seed activates the Gibbs free energy driving the adatoms easily heterogeneous deposition on seed surface. While in the presence of inert Ar atmosphere, there was no tendency observed for the adatoms to be deposited on the desired heterodimeric NPs. Therefore, $\mathrm{H}_{2}$ is reckoned to lower the energy barrier of Au nucleation and to induce more active sites over the seed FePd NPs. Hydrogen reduces the oxidation layer of the FePd surface to expose the Pd and Fe atoms. Meanwhile, the size effect of FePd seed NPs on the number of the Au domains has been investigated. 


\section{WILEY-VCH}
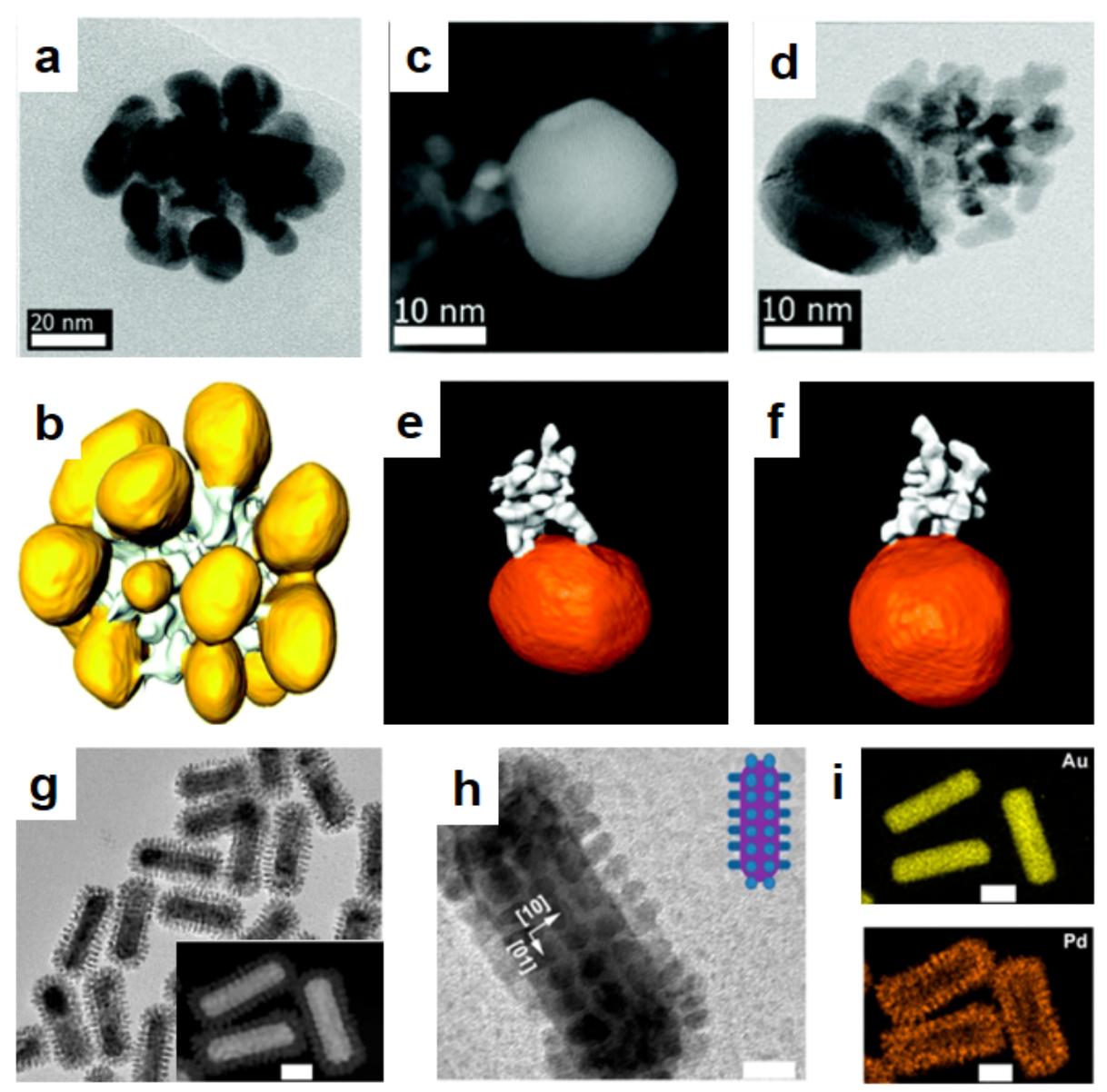

Figure 6. a-b) Single site growth mode assisted by PVP. c-f) Multiple sites growth side in the presence of CTAB. Reproduced with permission ${ }^{[52 a]}$. a-f) Copyright 2015, Royal of Society of Chemistry. g-i) Pd array-growth on the Au nanorods and corresponding EDX (Energy-Dispersive X-ray Spectroscopy) element mapping. Scale bars from (g) to (i) are $20 \mathrm{~nm}$. Reproduced with permission ${ }^{[54]}$. g-i) Copyright 2017, American Chemical Society.

With the size decreasing of FePd seed NPs, the number of Au nanodomains decreased due to the Ostwald ripening. Besides, reacting gas (e.g., $\mathrm{CO})$ is preferably adsorbed on the special facets of seeds which can promote the formation of plasmonic heteromers with different morphologies. ${ }^{[51 \mathrm{a}]}$ As described previously, the crystallinity property of seeds renders them capable as templates to induce the morphologies of final products. ${ }^{[37 \mathrm{c}]}$ The facets of Pd icosahedral NPs used as template promoted the formation of Pt domains with $\{100\}$ facets. ${ }^{104}$ In this case, CO cannot only play the role of reducing agent, but also of capping agent because of the oxidation process of $\mathrm{CO}$ conversion to $\mathrm{CO}_{2}$ on the selective Pt facets. Shape-directing of the secondary nanodomains on the seeds without 


\section{WILEY-VCH}

special facets of seeds has been achieved by Schlicke et al., who reported a cubic-like Pt tip-grown on the $\mathrm{CdS}$ nanorods (Figure $7 \mathrm{j}-1) \cdot{ }^{[56]} \mathrm{CO}$ possess stronger binding energy to $\{100\}$ facets rather than $\{111\}$ facets of $\mathrm{Pt}^{89}$
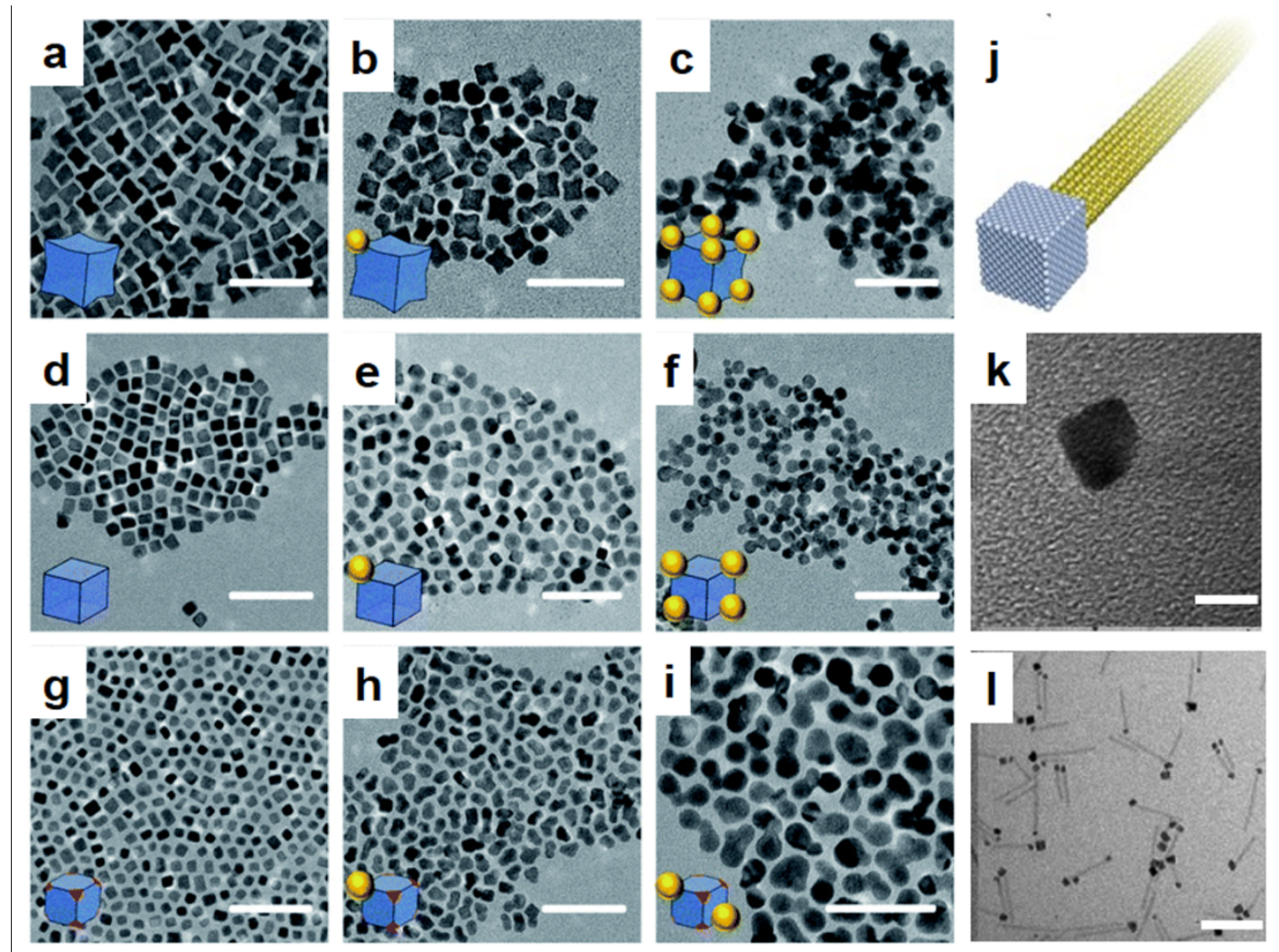

Figure 7. b), e), h) FePt-Au and c), f), i) FePt-Aun heteromers based on different sizedFePt seeds: a) $11 \mathrm{~nm}$, b) $8 \mathrm{~nm}$, g) $5 \mathrm{~nm}$, respectively. Reproduced with permission ${ }^{[55]}$. Scale bars in (a-i) are $50 \mathrm{~nm}$. (a-i) Copyright 2013, Royal of Society of Chemistry. j-1) Schematic synthesis of Pt cubic nanodomain at the tips of the semiconductor CdS NPs. Scale bars in (k) and (1) are $5 \mathrm{~nm}$ and $50 \mathrm{~nm}$, respectively. Reproduced with permission $^{[56]}$. j-1) Copyright 2013, Wiley-VCH.

Nanoframes, hollow and yolk-shell nanostructures with the higher surface area, high densities of low coordination atoms at the sites, exhibit miscible stability as well as enhanced catalytic and optical properties. ${ }^{[57]}$ The synthetic approach is related to galvanic replacement of more active metal elements of seeds or the underpotential deposition on the seed surface controlled by the thermodynamics and kinetics, as well as the Kirkendall effect taking place because of the difference of diffusing rate during the galvanic replacement or ions exchange process. ${ }^{[58]}$ During the galvanic reduction process, the Kirkendall effect may be coexisted or sequential acted on the formation of 


\section{WILEY-VCH}

hollow nanostructures, because the diffusion rate of adatoms into the nanocrystals and dissolution of the low underpotential atoms have an effect on their surface and morphology. ${ }^{[59]}$ In the first case, by merit of different reducing rate of for $\mathrm{Ag}^{+}$and $\mathrm{Cu}^{2+}$ because of their different electrochemical reduction potential, there will be sequential reducing rate of $\mathrm{Pt}$ and $\mathrm{Pd}$ on the surface of $\mathrm{AgCu}$ Janus seeds, which contributes to fabrication of Ag-Au-Pt-Cu Quadrometallic Janus NPs. ${ }^{[60]}$ In the second case, underpotential deposition of more active metal elements on the seed surface can decrease seeds reduction potential. Lutz et al. firstly prepared Au/Pd-Ag Janus seeds

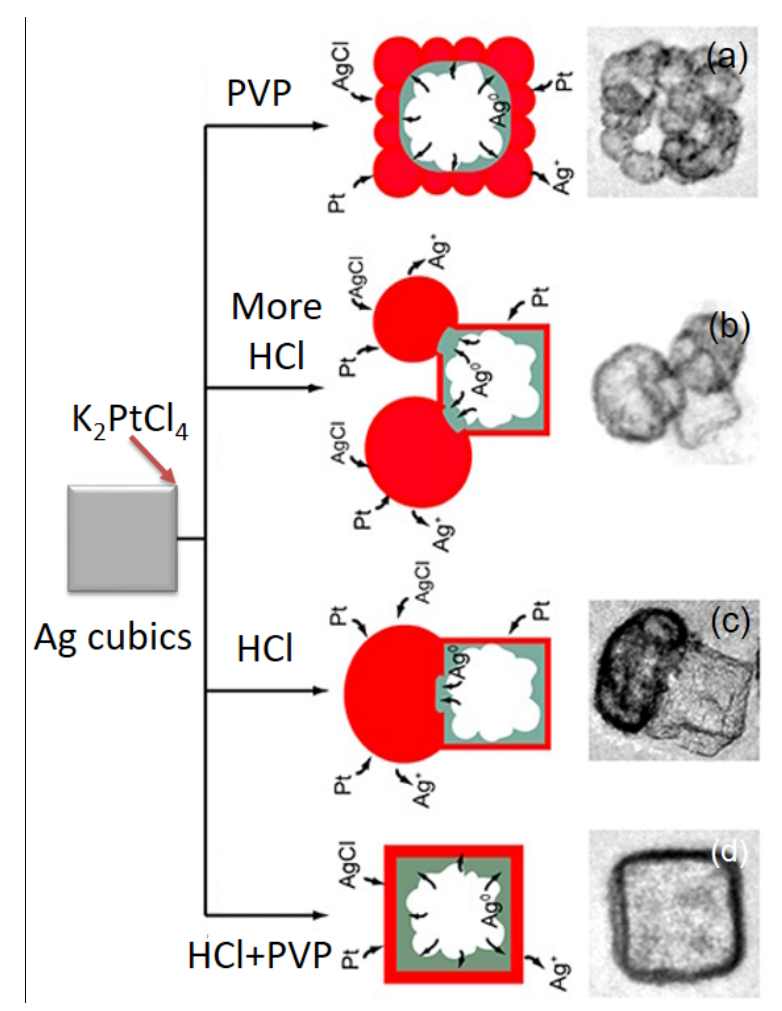

Figure 8. Schematic synthesis of a) popcorn, b) multimer, c) heterodimer, and d) nanobox Pt-Ag nanostructure. Reproduced with permission ${ }^{[61]}$. a-d) Copyright 2012, American Chemical Society.

by deposition of $\mathrm{Ag}$ on the $\mathrm{Au} / \mathrm{Pd}$ alloy seeds. Afterwards, they produced $\mathrm{Au} / \mathrm{Pd}-\mathrm{Ag}$ heteromeric nanostructures $(\mathrm{M}=\mathrm{Pt}, \mathrm{Au}, \mathrm{Pd})$ by the galvanic replacement reaction. The hollow nanodomains on the $\mathrm{Au} / \mathrm{Pd}-\mathrm{M}$ have increased the electrocatalytic performance for methanol oxidation. ${ }^{[62]}$ In another work, Yu et al. injected the silver and palladium precursors into the seed solution containing $\mathrm{Au}$ nanocrystals. The lower reduction 


\section{WILEY-VCH}

potential of Ag allows to be primarily and selectively deposited on the Au nanocrystals for the galvanic replacement with Pd precursors. As the concentration ratio of Ag and Pd increase, the growth mode is dominated by the Ag rather than Pd. Therefore, the shape of hybrids evolved to octahedron because the preferred exposure faces of Pd and $\mathrm{Ag}$ are $\{100\}$ and $\{111\}$ facets. ${ }^{[63]}$ Guo et al. demonstrated that $\mathrm{FeCl}_{3}$ can selectively lateral etch the Au@Ag core-shell nanorods to obtain Ag-tipped Au nanorods because of the lower concentration of CTAB at their lateral sides. Ag-tipped Au nanorods exhibit the higher catalytic activity due to their electronic effect and unique structure compared to Au@Ag core-shell nanorods. ${ }^{[64]}$ Zhang et al. have successfully obtained $\mathrm{Pd} / \mathrm{Ag}$ bimetallic hollow NPs by controlling the different amounts of $\mathrm{HCl}$ and capping agent, with a galvanic replacement-assisted method (Figure 8). ${ }^{61]}$ Firstly, PVP is easily adsorbed on Ag nanocubic seeds surface to tune their surface energy because of electronegative pyrrolidone rings and hydrophobic apolar groups, inducing the formation of $\mathrm{Ag} / \mathrm{Pt}$ nanoboxes (Figure 8d). By introducing $\mathrm{HCl}$, islands of insoluble $\mathrm{AgCl}$ immediately formed. The addition of $\mathrm{HCl}$ improve the production of $\mathrm{AgCl}$ grown on the $\mathrm{Ag}$ nanocubic seeds surface. Less $\mathrm{HCl}$ results in the formation of heterodimers (Figure 8c), while multimeric nanostructures form as the concentration of $\mathrm{HCl}$ increased (Figure 8b). The AgPt nanodomain prefers to heterogeneously grow at the tips of silver nanocubes because of the low-coordination atoms at the tips. Besides, a slight modification of $\mathrm{pH}$ value can tune the reaction kinetics. Because of both roles of $\mathrm{H}^{+}$and PVP, there would be a pattern of $\mathrm{AgCl}$ grown on the $\mathrm{Ag}$ nanocubic seeds surface. Therefore, popcorn-like nanostructures were created (Figure 8a). The hollow interior of secondary nanodomains can be further obtained by washing with the $\mathrm{NaCl}, \mathrm{Fe}\left(\mathrm{NO}_{3}\right)_{3}$ and $\mathrm{H}_{2} \mathrm{O}_{2}$.

\subsection{Plasmonic-semiconductors heteromers}

Semiconductors display the photoinduced electron transfer phenomenon. Under light irradiation, the hot electrons can be excited to its conduction band (CB) as well as left 


\section{WILEY-VCH}

hot holes in the valence band (VB). With this advantage, semiconductors have been at focus of study in the field of photocatalysis. There are several approaches for synthesis of plasmonic-semiconductor heteromers, involving (i) the heterogeneous growth of adatoms by photo-, chemical- reduction or thermodecomposition of plasmonic metallic precursors on the semiconductor seeds and (ii) the heterogeneous growth of adatoms by chemical reduction and thermal decomposition of semiconductor precursors on the plasmonic seeds. ${ }^{[65]}$ In particular, the photoinduced electrons can reduce the secondary metal precursors for the epitaxial growth. For example, Ag precursors have been reduced on the amino group-functionalized carbon dots (CD) under light irradiation, thereby forming CD-Ag heterodimers. ${ }^{[66]}$ In the latter case, Li et al. have found that the low surface energy barrier and lattice mismatch between $\mathrm{Au}$ and $\mathrm{ZnO}$ can promote the heterogeneous of $\{101\}$ facets of $\mathrm{ZnO}$ on the $\mathrm{Au}$ seeds for the formation of $\mathrm{Au}-\mathrm{ZnO}$ pyramids heteromers. The $\{0001\}$ planes of $\mathrm{ZnO}$ pyramids are contributed to the polar/metal interface. ${ }^{[67]}$

However, the faster recombination of hot-electrons and charges in semiconductor, as well as excited photons with higher excited energy inhibit the application of semiconductor. With LSPR of plasmonic metal NPs, hot electrons can be transferred to the conduction band of semiconductor which enlarge the life-time of hot electrons. Additionally, the excited photons can extend to the vis-NIR range. So plasmonsemiconductor hybrid NPs have been intensively studied, especially, plasmonicsemiconductor heteromers. ${ }^{[68]}$ Han and colleagues have developed a series of $\mathrm{Au}-\mathrm{TiO}_{2}$ heteromers through carefully tuning the interfacial energy and elastic energy. The Au$\mathrm{TiO}_{2}$ Janus heteromers were produced by only injecting $3 \mathrm{ml}$ of $\mathrm{TiO}_{2}$ precursors. Once the $3 \mathrm{ml}$ of $\mathrm{TiO}_{2}$ precursors are averagely added by 3 separate times, the eccentric Au$\mathrm{TiO}_{2}$ hybrids are obtained. These two asymmetric nanostructures lead to the elastic energy increase. More $\mathrm{TiO}_{2}$ precursors were injected leading to the formation of $\mathrm{Au}-$ $\mathrm{TiO}_{2}$ concentric nanostructures. ${ }^{[69]}$ 


\section{WILEY-VCH}

In order to achieve more complex heterostructures, decreasing the big lattice mismatch between noble metal and semiconductor is key point by releasing the lattice strain and Gibbs free energy. Weng et al. have tuned the surface energies through surface pre-modification of seeds with Ag before the overgrowth process of adatoms on seeds. ${ }^{[70]} \mathrm{Ag}$ is chosen as the intermediate layer material for selective growth of highorder frameworks due to the following reasons: firstly, Ag can selectively nucleate and grow on the designed sites and faces of seeds. In addition, the high acid softness and electronegativity of $\mathrm{Ag}$ (similar to many chalcogenide anions) facilitate various chemical transformations, including the galvanic replacement of metals and cation exchange of compounds. ${ }^{[71]}$ As shown in Figure 9a, they have fabricated one common intermediate Ag layer on starting seeds surface, based on the Lewis acid-base reaction mechanism via ion exchange. ${ }^{[70,72]}$ Figure 9a shows the schematic synthesis of plasmonic heteromeric nanostructures by the intermediate Ag layer. when Ag is added to an existing subunit $\alpha$ (stage I), only two possible geometries can occur: core-shell (Route 1) and dimeric (Route 2) modalities (stage II). Both Route 1 and Route 2 can lead to a subunit $\alpha$-amorphous $\mathrm{Ag}_{2} \mathrm{X}$ intermediate heterodimer (stage III). A targeted subunit $\beta$, can be achieved by ionic exchange of $\mathrm{Ag}_{2} \mathrm{X}$ with metal ions (stage IV). By carefully controlling the number of Ag nanodomains growth on the surface of the Pt nanocubic seeds, they were able to obtain a series of higher-order heteromers (Figure 9b-g). In this way, ternary and oligomer hybrid NPs are synthesized based on the heterodimeric seeds creating more functionalities. ${ }^{[73]}$ Liang and co-workers have demonstrated a simple and versatile method for generating $\mathrm{Au}-\mathrm{AgCdSe}$ hybrid nanorods with well controlled morphologies and symmetries. ${ }^{[74]}$ They firstly tune the geometry of Ag shell on the Au the gold nanorods by the different $\mathrm{pH}$ value at $30{ }^{\circ} \mathrm{C}$. Two types of Au-Ag hybrid NPs have been produced in this step, one is at $\mathrm{pH}$ value of 6.8 , another type is at $\mathrm{pH}$ value of 8.6. More silver was reduced at the tips of Au nanorods seeds surface at a higher $\mathrm{pH}$ value because the reducing power of glycine is sensitive to the $\mathrm{pH}$ value. Afterwards, 


\section{WILEY-VCH}

selenization of $\mathrm{Ag}$ and cation exchanges of $\mathrm{Cd}^{2+}$ on $\mathrm{Au}$ nanorods occurs at $90^{\circ} \mathrm{C}$ in the presence of $\mathrm{Cd}^{2+}$, Se, and $\mathrm{NaBH}_{4}$. The reducing ability of $\mathrm{NaBH}_{4}$ also is sensitive to $\mathrm{pH}$ value. After carefully controlling the $\mathrm{pH}$ value in the second step, three distinct heteromeric nanostructures of $\mathrm{Au}-\mathrm{AgCdSe}$ were produced respectively, mike-like ( $\mathrm{pH}$ value of 7.2), dumbbell-like ( $\mathrm{pH}$ value of 9.8), and toothbrush-like nanorods ( $\mathrm{pH}$ value of 8.6) (Figure 9h-j).

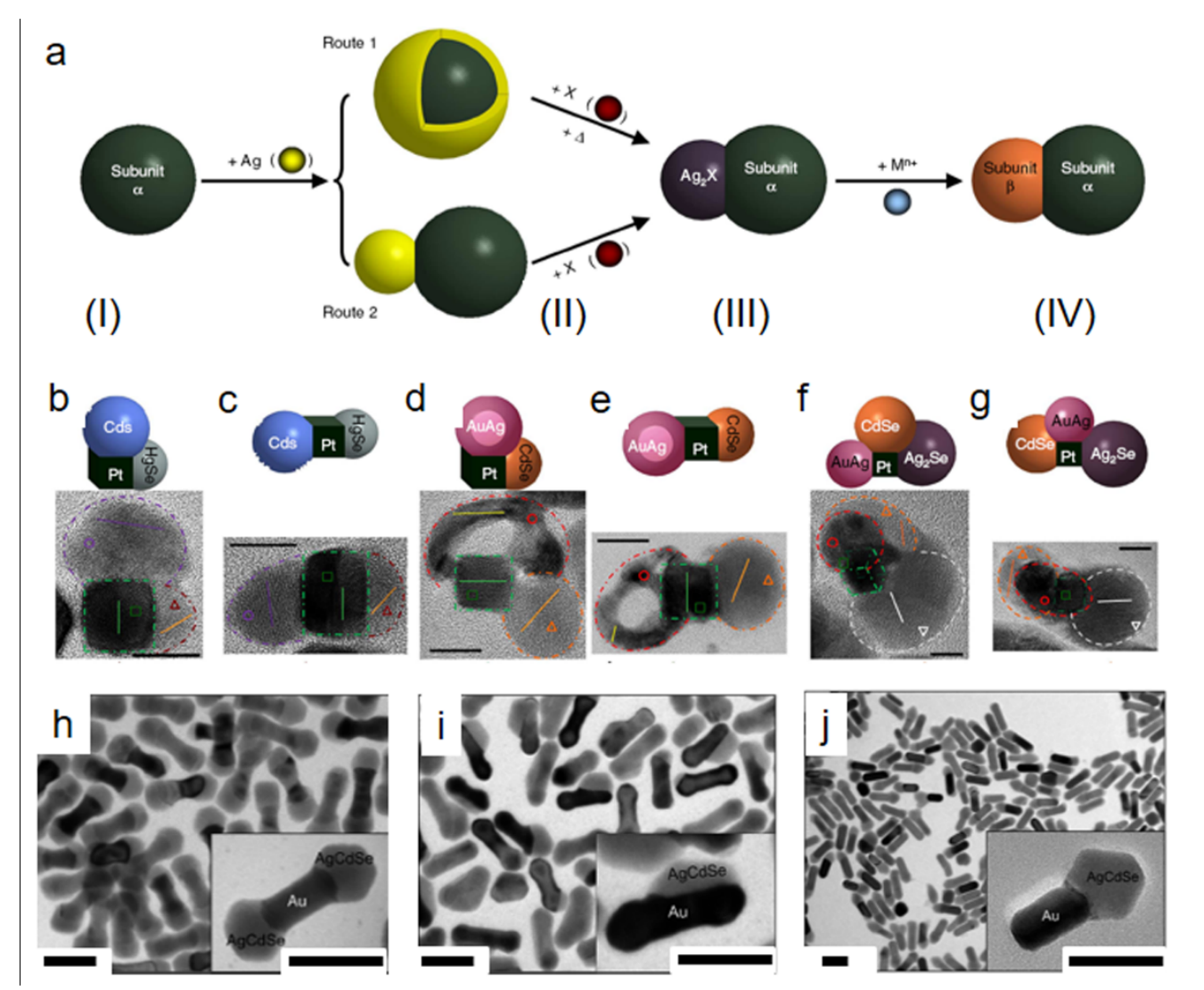

Figure 9. a) Schematic synthesis for precise engineering the higher-order heteromers through tuning the surface energy by a layer of Ag. When Ag is added to an existing subunit $\alpha$ (stage I), only two possible geometries can occur: core-shell (Route 1) and dimeric (Route 2) conformations (stage II). Both Route 1 and Route 2 can lead to a subunit $\alpha$-amorphous $\mathrm{Ag}_{2} \mathrm{X}$ intermediate heterodimer (stage III). A targeted subunit $\beta$, $\mathrm{M}_{2}{ }^{\mathrm{n}+} \mathrm{X}_{\mathrm{n}}$, can be achieved by ionic exchange of $\mathrm{Ag}_{2} \mathrm{X}$ with metal ions of $\mathrm{M}^{\mathrm{n}+}$ (stage IV). b-g) Higher-order heteromers: b,c)Pt-CdS-HgSe, d,e)Pt-AuAg-CdSe and f,g) Pt-Ag 2 SeAuAg-CdSe. Scale bars in (b-g) are $50 \mathrm{~nm}$. Reproduced with permission ${ }^{[70]}$. a-g) Copyright 2014, Nature Publishing Group. h) dumbbell-like and i) toothbrush-like $\mathrm{Au}-\mathrm{AgCdSe}$ nanorods. j) the mike-like $\mathrm{Au}-\mathrm{AgCdSe}$ nanorods. Reproduced with permission $^{[74]}$. h-j) Copyright 2012, American Chemical Society. Scale bars in (h-j) are $50 \mathrm{~nm}$.

\subsection{Plasmonic-magnetic heteromers}




\section{WILEY-VCH}

Plasmonic-magnetic heteromers combining both type of properties have been broadly applied in the biomedical, catalysis and optoelectronics applications. ${ }^{[75]}$ To obtain plasmonic-magnetic heteromers should overcome their limited water stability and larger mismatch. Typically, there are two directions for the synthesis of these plasmonic- $\mathrm{Fe}_{\mathrm{x}} \mathrm{O}_{\mathrm{y}}$ heterostructures, including (i) the heterogeneous nucleation and growth of gold adatoms on the $\mathrm{Fe}_{\mathrm{x}} \mathrm{O}_{\mathrm{y}}$ seeds surface at suitable temperature and (ii) the thermal decomposition of iron precursors at high temperature on the Au seeds surface. ${ }^{[76]}$ In both cases, these heteromeric nanostructures are produced after the thermal decomposition of a secondary metallic precursor over the seed surface at a given temperature. The lattice mismatch (around 2.9\%) between plasmonic and $\mathrm{Fe}_{\mathrm{x}} \mathrm{O}_{\mathrm{y}}$ results in mechanical stress. Tancredi et al. investigated the effect of size of $\mathrm{Au}$ seeds on the final morphologies of $\mathrm{Au}-\mathrm{FeO}_{\mathrm{x}}$ heteromers. $3 \mathrm{~nm}$ Au NPs applied as seeds favour the synthesis of heterodimers while 8 $\mathrm{nm} \mathrm{Au} \mathrm{NPs} \mathrm{used} \mathrm{as} \mathrm{seeds} \mathrm{prefer} \mathrm{to} \mathrm{form} \mathrm{flower-like} \mathrm{heteromers,} \mathrm{which} \mathrm{is} \mathrm{mainly}$ contributed to the polycrystalline and multi-twinned nature of $8 \mathrm{~nm} \mathrm{Au} \mathrm{NPs.}{ }^{[77]}$

In order to overcome the mechanical stress, Jiang et al. developed one facile approach where oxidation of Fe occurs on the surface Au-Fe Janus seeds resulting in the production of $\mathrm{Au}-\mathrm{Fe}_{3} \mathrm{O}_{4}$ heteromers. ${ }^{[78]}$ The mechanical stress increases as size of $\mathrm{Au}$ domains increases, as a result of $\mathrm{Au}$ being detached from seeds. ${ }^{[76 a, 79]}$ As shown in Scheme 1 in Figure 10, mechanical stress was released by virtue of a thin layer of amorphous iron oxide formation on Fe seeds, which direct the heterogeneous nucleation and growth of Ag domains. With Ostwald ripening, small silver NPs become larger. ${ }^{[80]}$ As reported by Peng et al. in Figure 10a, silver salts were reduced to several smaller Ag NPs on $\mathrm{Fe} @ \mathrm{Fe}_{\mathrm{x}} \mathrm{O}_{\mathrm{y}}$ immediately, because of the lower interfacial energy between Ag and $\mathrm{Fe}_{\mathrm{x}} \mathrm{O}_{\mathrm{y}}$ shell. Meanwhile, Ostwald ripening mechanism took place along with sufficient growth time leading to several smaller Ag NPs grown to larger Ag domains step by step (Figure 10b-f). The size of Ag domains was also controlled by the injection amount of 


\section{WILEY-VCH}

the silver precursors. However, more injection of silver salts can increase surface free energy of $\mathrm{Ag}^{0}$, resulting in its homogeneous nucleation and growth. It is noteworthy that

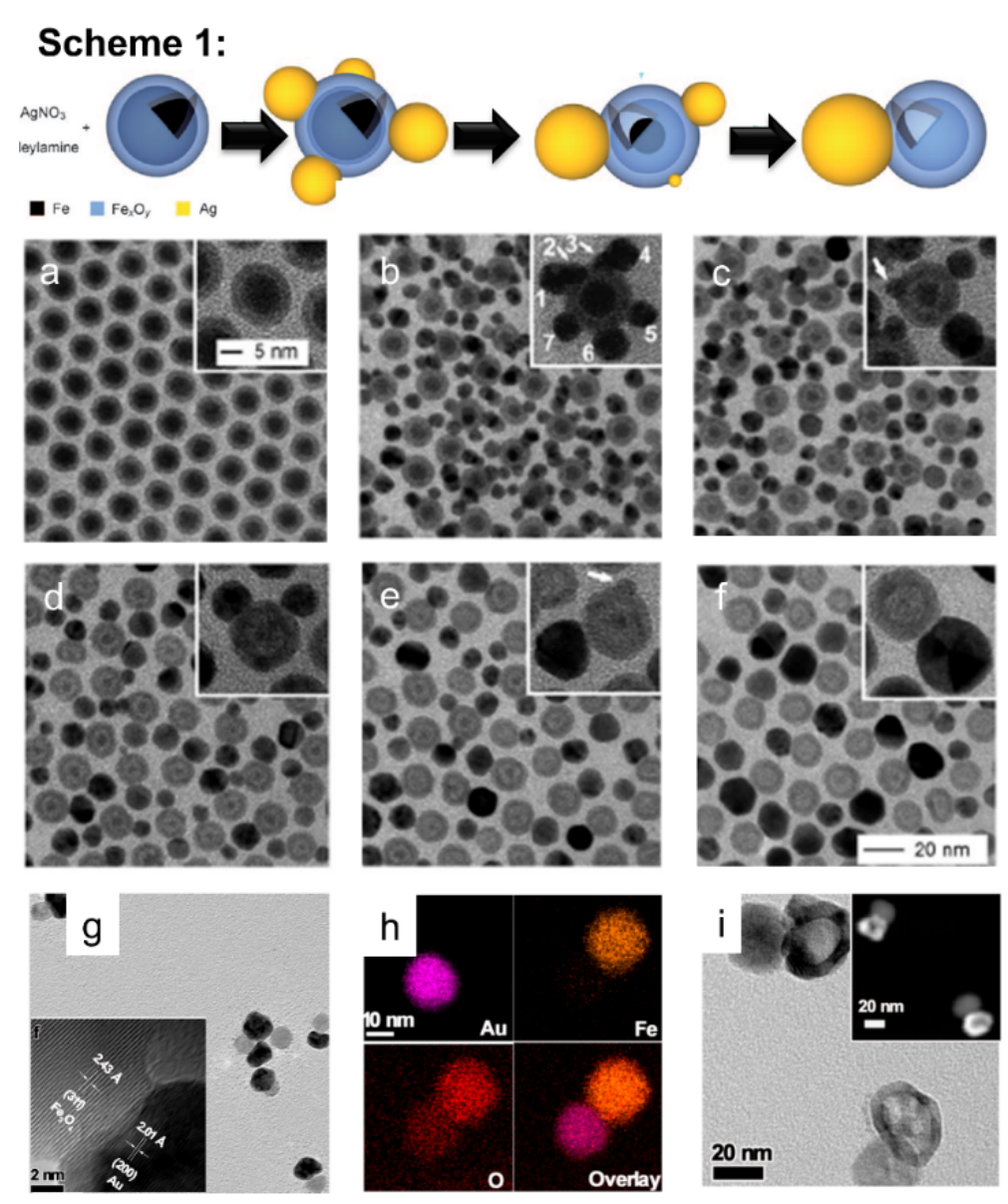

Figure 10. Scheme 1: Synthesis of the Ag-Fe@ $\mathrm{Fe}_{\mathrm{x}} \mathrm{O}_{\mathrm{y}}$ heteromers by tuning their lattice mismatch. TEM images of $\mathrm{Ag}-\mathrm{Fe} @ \mathrm{Fe}_{\mathrm{x}} \mathrm{O}_{\mathrm{y}}$ heteromers evolution from: a) 0, b) $2 \mathrm{~s}$, c) 30 s, d) $90 \mathrm{~s}$, e) $180 \mathrm{~s}$, and f) $300 \mathrm{~s}$. Reproduced with permission ${ }^{[80]}$. a-f) Copyright 2012, American Chemical Society. g) Hydrophilic Au-Fe ${ }_{3} \mathrm{O}_{4}$ heteromers and h) corresponding EDX element mapping. i) Hydrophilic Ag@ $\mathrm{Au}_{\text {hollow }}-\mathrm{Fe}_{3} \mathrm{O}_{4}$ heteromers. Reproduced with permission ${ }^{[81]}$. g-i) Copyright 2019, American Chemical Society.

those plasmonic-magnetic heteromers are often produced in the presence of hydrophobic ligands (oleylamine or oleic acid). 1-octadecene is one of the most frequently used solvents for synthesis of heteromeric nanostructures in the hydrophobic phase due to its high boiling point and higher viscosity kinematic. High viscosity can decrease the Gibbs free energy of the system which can promote the heterogeneous nucleation and growth of adatoms on the seeds. Recently, Zeng et al. have prepared hydrophilic plasmonicmagnetic heterodimeric NPs in the presence of sodium citrate as weaker reducing agent 


\section{WILEY-VCH}

(Figure 10g-i). Firstly, they exchanged the surface ligand of seeds from oleic acid to hydrophilic ligand. The presence of $\mathrm{Fe}^{2+}$ on the surface of seeds with reductive property promoted the reduction and deposition of gold salts to Au NPs. The inset in Figure $10 \mathrm{~g}$ reveals the lattice were coherent at solid-solid interface. Furthermore, $\mathrm{Fe}_{3} \mathrm{O}_{4}-\mathrm{Ag} @ \mathrm{Au}$ hollow heteromers were produced (Figure 10i) when $\mathrm{Fe}_{3} \mathrm{O}_{4}-\mathrm{Ag}$ were used as seeds.

The active sites of seeds can also show catalytic function and electrons transfer. ${ }^{[82]}$ Choi et al. prepared FePt-Au heterodimers with superparamagnetic behavior in the aqueous solution. The Au precursors are reduced on the FePt surface in the presence of $\mathrm{H}_{2}$ where FePt acted as the catalytic sites. ${ }^{[83]}$ Lim et al. have illustrated that the formation of $\mathrm{Pt}-\mathrm{Au}$ heterodimers is conducted by the slow injection rate of $\mathrm{Au}$ precursors into $\mathrm{Pt}$ seed solution at room temperature. ${ }^{[84]}$ Besides, Pt served as catalytic sites can accelerate the heterogeneous nucleation of $\mathrm{Au}$ which is the key point for the room temperature epitaxial synthesis. Heteromers between $3 \mathrm{~d}$ transition metal and noble metal NPs are not easily prepared because of their larger lattice mismatch. Recently, this situation was challenged as Pt-tip-on Au nanorods were applied as seeds which promoted the reduction of Ni adatoms..$^{[3,85]}$ Nevertheless, Buck et al. achieved the heterogeneous nucleation of a third metallic element onto the electronegative $\mathrm{Pt}$ sites of $\mathrm{Pt}_{-} \mathrm{Fe}_{3} \mathrm{O}_{4}$ seeds (Figure 11a) and a fourth metallic element onto the $\mathrm{Au}-\mathrm{Pt}-\mathrm{Fe}_{3} \mathrm{O}_{4}$ seeds (Figure 11b). ${ }^{[86]}$ The TEM images of $\mathrm{Pt}$ and $\mathrm{Pt}-\mathrm{Fe}_{3} \mathrm{O}_{4} \mathrm{NPs}$ have been shown in Figure $11 \mathrm{c}$ and $\mathrm{d}$. In the $\mathrm{Pt}-\mathrm{Fe}_{3} \mathrm{O}_{4}$ Janus seeds, the downward band bending of $\mathrm{Fe}_{3} \mathrm{O}_{4}$ reveals there is charge accumulation via the solid-solid nanointerface. ${ }^{[87]}$ Electronic communication among the domains of heteromers via solid-solid interface leads to the electronic disbalance on the surface of dimers. ${ }^{[22 b, 28 f]}$ So, there are richer electrons distribution on Pt domains where electrons are drawn from the $\mathrm{Fe}_{3} \mathrm{O}_{4}$ nanodomains via solid-solid nanointerface. ${ }^{[86]}$ Thus, adatoms can selectively nucleate on the special sites of the $\mathrm{Pt}-\mathrm{Fe}_{3} \mathrm{O}_{4}$ and $\mathrm{Au}-\mathrm{Pt}-\mathrm{Fe}_{3} \mathrm{O}_{4}$ hybrid NPs for the desired morphology (Figure 11a,b). Typical TEM images are shown in Figure 11e-i. Additionally, sulfur species were used as adhesives for synthesis of trimers and 


\section{WILEY-VCH}

oligomers through their different chemical reactivities with metal atoms. ${ }^{[88]}$ Sulfur atoms also preferred to attach on $\mathrm{Au}$ rather than $\mathrm{Cu}, \mathrm{Pt}, \mathrm{Fe}_{3} \mathrm{O}_{4}$, because the reactivity and

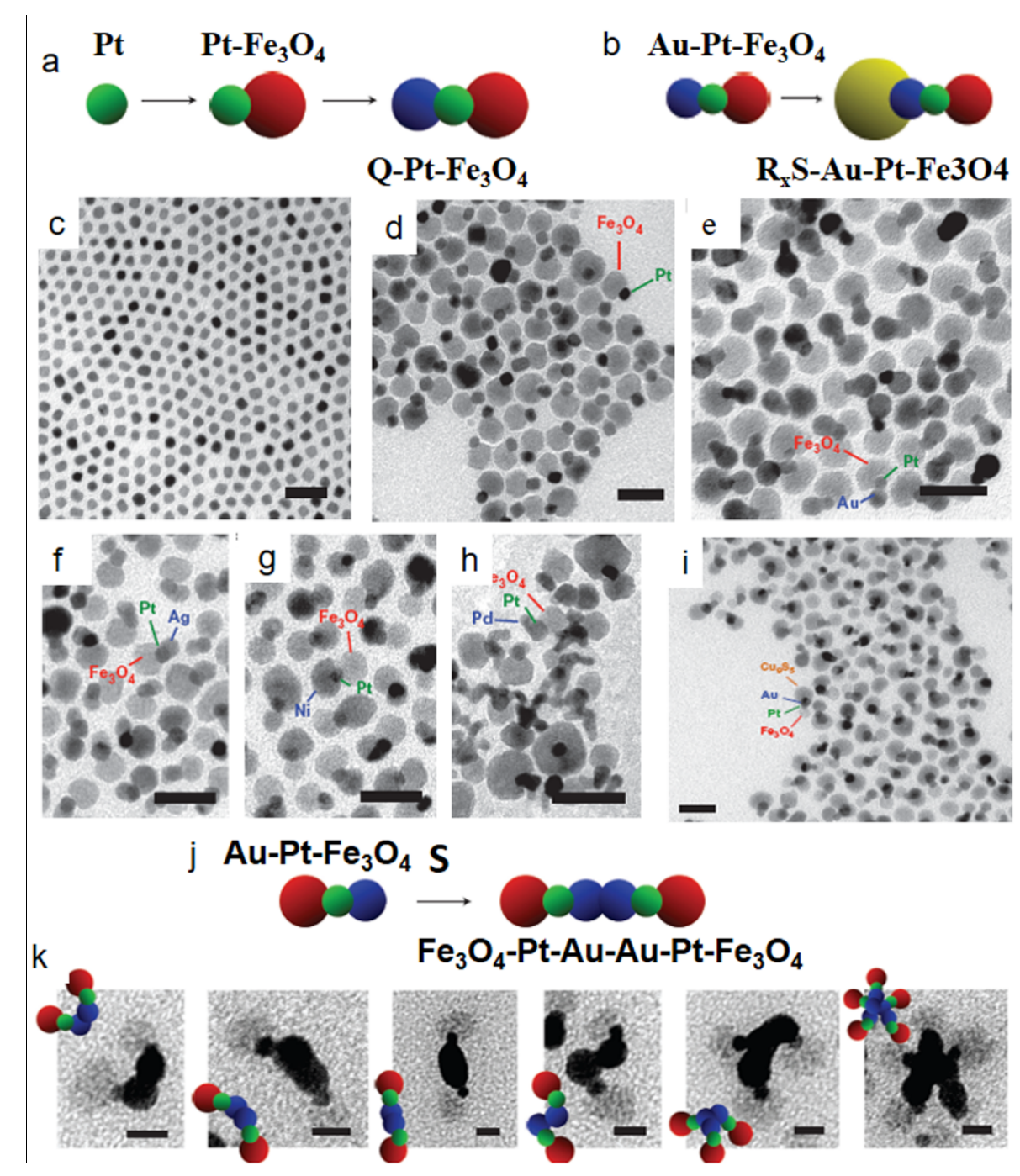

Figure 11. a) metal adatoms $\mathrm{Q}(\mathrm{Q}=\mathrm{Ag}, \mathrm{Au}, \mathrm{Ni}, \mathrm{Pd})$ deposition on the electrical negative $\mathrm{Pt}$ domains to form X-Pt-Fe $\mathrm{O}_{4}$ heteromers. c) Pt seeds, d) $\mathrm{Pt}_{-} \mathrm{Fe}_{3} \mathrm{O}_{4}$ binary heteromers, e) $\mathrm{Au}-\mathrm{Pt}-\mathrm{Fe}_{3} \mathrm{O}_{4}$, f) $\mathrm{Ag}-\mathrm{Pt}-\mathrm{Fe}_{3} \mathrm{O}_{4}$, g) Ni-Pt-Fe $\mathrm{O}_{4}$, and h) $\mathrm{Pd}-\mathrm{Pt}-\mathrm{Fe}_{3} \mathrm{O}_{4}$ ternary heteromers. b) $\mathrm{R}_{\mathrm{x}} \mathrm{S}_{\mathrm{y}}-\mathrm{Au}-\mathrm{Pt}-\mathrm{Fe}_{3} \mathrm{O}_{4}$ hybrid tetramers $(\mathrm{R}=\mathrm{Cu}, \mathrm{Pb})$ based on ternary heteromeric seeds. (I) $\mathrm{Cu}_{9} \mathrm{~S}_{5}-\mathrm{Au}-\mathrm{Pt}-\mathrm{Fe}_{3} \mathrm{O}_{4}$ oligomers. j) Higher-order heteromers based on $\mathrm{Au}-\mathrm{Pt}-\mathrm{Fe}_{3} \mathrm{O}_{4}$ ternary seeds. k) Linear and bent higher-order branched $\mathrm{Fe}_{3} \mathrm{O}_{4}-\mathrm{Pt}-\mathrm{Au}-\mathrm{Au}-\mathrm{Pt}-\mathrm{Fe}_{3} \mathrm{O}_{4}$ nanostructures. Scale bars in (a-i) are $25 \mathrm{~nm}$ and Scale bar in (k) is $10 \mathrm{~nm}$. Reproduced with permission ${ }^{[86]}$. a-k) Copyright 2012, Nature Publishing Group.

mobility of atomic sulfur species on Au compared to other metal salts is larger. When $\mathrm{AuCu}$ alloy or PtCu alloy NPs were used as seeds, sulfur preferred to drive $\mathrm{Cu}$ atoms to the surface resulting in the $\mathrm{Au}-\mathrm{Cu}_{2} \mathrm{~S}$ and $\mathrm{Pt}-\mathrm{Cu}_{2} \mathrm{~S}$ heteromer NPs. Furthermore, $\mathrm{Ag}_{2} \mathrm{~S}$ hollow interiors Pt NPs $\left(\mathrm{Ag}_{2} \mathrm{~S}-\mathrm{hPt}\right)$ have been generated depending on the different 


\section{WILEY-VCH}

chemical reactivity between the $\mathrm{S}^{2-}$ ions and $\mathrm{Pt}$ as well as $\mathrm{Ag}$ metal elements of the $\mathrm{Ag} @ \mathrm{Pt}$ core-shell NPs. ${ }^{\left[{ }^{[9]}\right]}$ Therefore, $\mathrm{Cu}_{\mathrm{y}} \mathrm{S}_{\mathrm{x}}-\mathrm{Au}-\mathrm{Pt}-\mathrm{Fe}_{3} \mathrm{O}_{4}$ oligomers were obtained in the presence of sulfur when $\mathrm{Au}-\mathrm{Pt}-\mathrm{Fe}_{3} \mathrm{O}_{4}$ were used as seeds. Besides, Au exhibited higher electronegativity on the surface of the $\mathrm{Au}-\mathrm{Pt}-\mathrm{Fe}_{3} \mathrm{O}_{4}$ hybrid trimers NPs, so sulfur was used as the mucilage glue to fuse $\mathrm{Au}-\mathrm{Fe}_{3} \mathrm{O}_{4}, \mathrm{Au}-\mathrm{Pt}-\mathrm{Fe}_{3} \mathrm{O}_{4}$ leading to the high order frameworks of $\mathrm{Fe}_{3} \mathrm{O}_{4}-\mathrm{Au}-\mathrm{Fe}_{3} \mathrm{O}_{4}$ and $\mathrm{Fe}_{3} \mathrm{O}_{4}-\mathrm{Pt}-\mathrm{Au}-\mathrm{Pt}-\mathrm{Fe}_{3} \mathrm{O}_{4}$ (Figure $11 \mathrm{j}-\mathrm{k}$ ).

\section{Properties and applications of plasmonic heteromeric superstructures}

Based on LSPR property of plasmonic domains, plasmonic-M ( $\mathrm{M}=$ noble metal, semiconductor, magnetic NPs) heteromers can display "all-in-one" activities that simultaneously present all the different range of functionalities of each domain. Besides, plasmonic heteromers can also demonstrate new properties and possess improved properties in comparison to those of their components. With the advantages of structure effect, plasmonic heteromers, possessing LSPR and other properties together via solidsolid nanointerface, have been applied in numerous fields, including photocatalysis, ${ }^{[90]}$ electrocatalysis, ${ }^{[52 a, 91]}$ optical sensing, ${ }^{[92],[93]}$ biomedical imaging and cancer therapy. ${ }^{[75 b \text {, }}$ 94],[95] 


\section{WILEY-VCH}

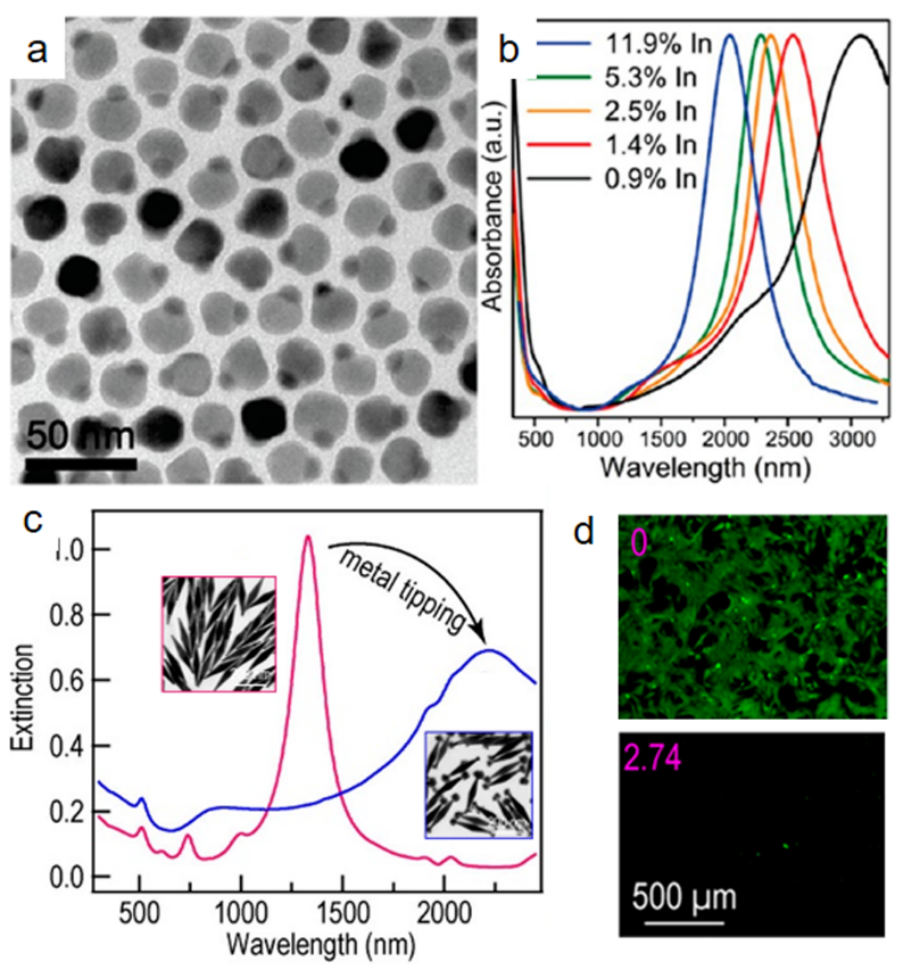

Figure 12. a) and (b) are TEM images and vis-NIR absorption spectra of Au-ICO heteromers. Reproduced with permission ${ }^{[84]}$. Copyright 2014, American Chemical Society. (c) vis-NIR spectra of $\mathrm{Au}$ nanopyramids and $\mathrm{AgPd}$ tipped-Au nanopyramids. The insets are their corresponding TEM images. (d) The U-87 MG cells are treated with different power $(0$ and $2.74 \mathrm{~W}$ ) of lasers in the presence of silica-coated AgPd tipped-Au nanopyramids. Reproduced with permission ${ }^{[96]}$. Copyright 2017, American Chemical Society.

So far, LSPR of plasmonic heteromer NPs can be localized at visible-NIR region, which can meet the needs of the second water window and infrared nanophotonics applications. In contrast to plasmonic heteromer NPs, the LSPR tuning of plasmonic metal NPs can only be controlled by their aspect ratio and size. For example, the LSPR of $\mathrm{Au}$ NRs are ranged from $600 \mathrm{~nm}$ to $1000 \mathrm{~nm}$, as well as the LSPR of Au nanoprisms are extended to $1300 \mathrm{~nm}$. On the other hand, the composition of Au/Ag alloy nanoparticles or plasmonic hybrid NPs also affect their LSPR. For example, the LSPR of AgAu hollow nanostructures are controlled by the injection amount of gold precursors. As gold salts are injected into the $\mathrm{Au} / \mathrm{Ag}$ nanorods solution, the LSPR will be red-shift because of the formation of Au NRs-AuAg yolk-shell nanostructures. Several scientists have developed the $\mathrm{Au}-\mathrm{SiO}_{2},{ }^{[97]} \mathrm{Au}-\mathrm{MOF}$, ${ }^{[97]}$ and $\mathrm{Au}-\mathrm{Ag}$ core-shell or janus $\mathrm{NPs}^{[98]}$ to tune their LSPR property, since the LSPR of plasmonic metal NPs is quite sensitive to 


\section{WILEY-VCH}

varies of the dielectric function of surround environment materials. Recently, the controllability of structures and compositions of plasmonic heteromers is one effective route to tune the LSPR to near-infrared region. For example, Au-indium doped cadmium oxide (ICO) heterodimers possess the dual LSPR peak in the visible and mid-IR region, the dual LSPR peaks are discerned to be induced by the Au and ICO, respectively. ${ }^{[84]}$ The lattice fringes are approached to the coherent nanointerface, thereby, the overgrowth of ICO on Au nanoseeds are modulated for the obtaining of Au-ICO heteromers. Their TEM iamges are shown in the Figure 12a. The LSPR of plasmonic nanocrystals is conducted by their fixed carrier, while the doping degree of ICO can tune the imaginary part of their dielectric function. In the Figure $12 \mathrm{~b}$, The wavelength of maximum LSPR intensity can be adjusted from 1.9 to $3.6 \mu \mathrm{m}$ by varying the composition ratio of indium in ICO nanodomains. ${ }^{[84]}$ Additionally, LSPR properties of plasmonic metal NPs can endow the other noble metal NPs plasmonic properties for nanophotonic applications. Zhu et al. have deposited one small AgPd nanodomains at the tips of Au nanobipyramids assisted by the Ag sacrificed template, which largely tunes the LSPR in the entire NIR region, from $\sim 1330 \mathrm{~nm}$ to $2216 \mathrm{~nm}$. The tip-overgrowth of AgPd nanodomains on $\mathrm{Au}$ nanobipyramids are realized under the synergistic effect of galvanic replacement reduction between $\mathrm{Ag}(0)$ and $\mathrm{Pd}(2+)$, co-reduction of $\mathrm{Ag}(+)$ and $\mathrm{Pd}(2+)$ by ascorbic acid and CTAB surfactants. In the Figure 12c, small AgPd nanodomains are localized at the tips of Au nanobipyramids induce the LSPR wavelength largely red shift with near $900 \mathrm{~nm}$ because of the large imaginary part of the Pd components. The silica coating AgPd-tipped $\mathrm{Au}$ nanopyuramids can overcome the second biological transparency window leading to high photothermal therapy efficiency. In the Figure 12f, There are nearly no fluorescence marked cancer cells after the laser irradiation.

Plasmonic domains of heteromers not only possess tunable LSPR properties which can harvest light, but also create the Schottky junction cross the solid-solid nanointerface enabling the conversion improvement of solar energy into chemical energy. There are 


\section{WILEY-VCH}

two main energy transfer mechanisms for plasmon-enhanced photocatalysis, including hot electron injection and plasmon-enhanced electromagnetic field. Under the laser irradiation, a pair of electrons and holes will be generated on the metal NPs surface. On one hand, plasmonic heteromeric nanostructure will improve separation efficiency via two routes. One is the indirectly electron transfer that hot electrons migrate into the adsorbed electrons acceptors (adsorbed molecules, semiconductor, ${ }^{[99]} \mathrm{Pd}^{[20]} \mathrm{Pt}^{[47 \mathrm{e}]}$ ), while the other one is the directly electron transfer that hot electrons migrated into the hybridization states of plasmonic nanocrystals and adsorbed acceptors. Additionally, the enhanced localized electromagnetic field has increased the yields of hot electrons-holes pairs thousand times in the theoretical simulations. The $\mathrm{Au}$ nanochains $/ \mathrm{Zn}_{0.67} \mathrm{Cd}_{0.33} \mathrm{~S}$ hybrid NPs have possessed 3.5 times photocatalytic efficiency compared to isolated Au nanoparticles, because of the "hot spots" in the Au nanochains have boosted the generation of hot electrons. ${ }^{[100]}$ In contrast to core-shell nanostructures, it is convenient for the heteromers to access the electron donor to refill the sacrificed electrons on the plasmonic metal surface and low-energy decreasing. Hot charges generated from Landau damping of LSPR have been used to promote photochemical reactions, such as hydrogen evolution reaction, $\mathrm{CO}_{2}$ reduction and Suzuki-Miyaura coupling reaction. ${ }^{[101]}$ Compared to damping or vanished LSPR for Au-Pd core-shell nanostructures, Au-Pd plasmonic heteromers still do present a clear LSPR peak (Figure 13a). ${ }^{[102]}$ Under laser irradiation, transient absorption spectra in Figure $13 \mathrm{~b}$ revealed that $\mathrm{Au}-\mathrm{Pd}$ superstructures experienced longer decay time of hot electrons because of their larger solid-solid interface compared to other hybrid nanostructures. Therefore, the efficiency of hot 

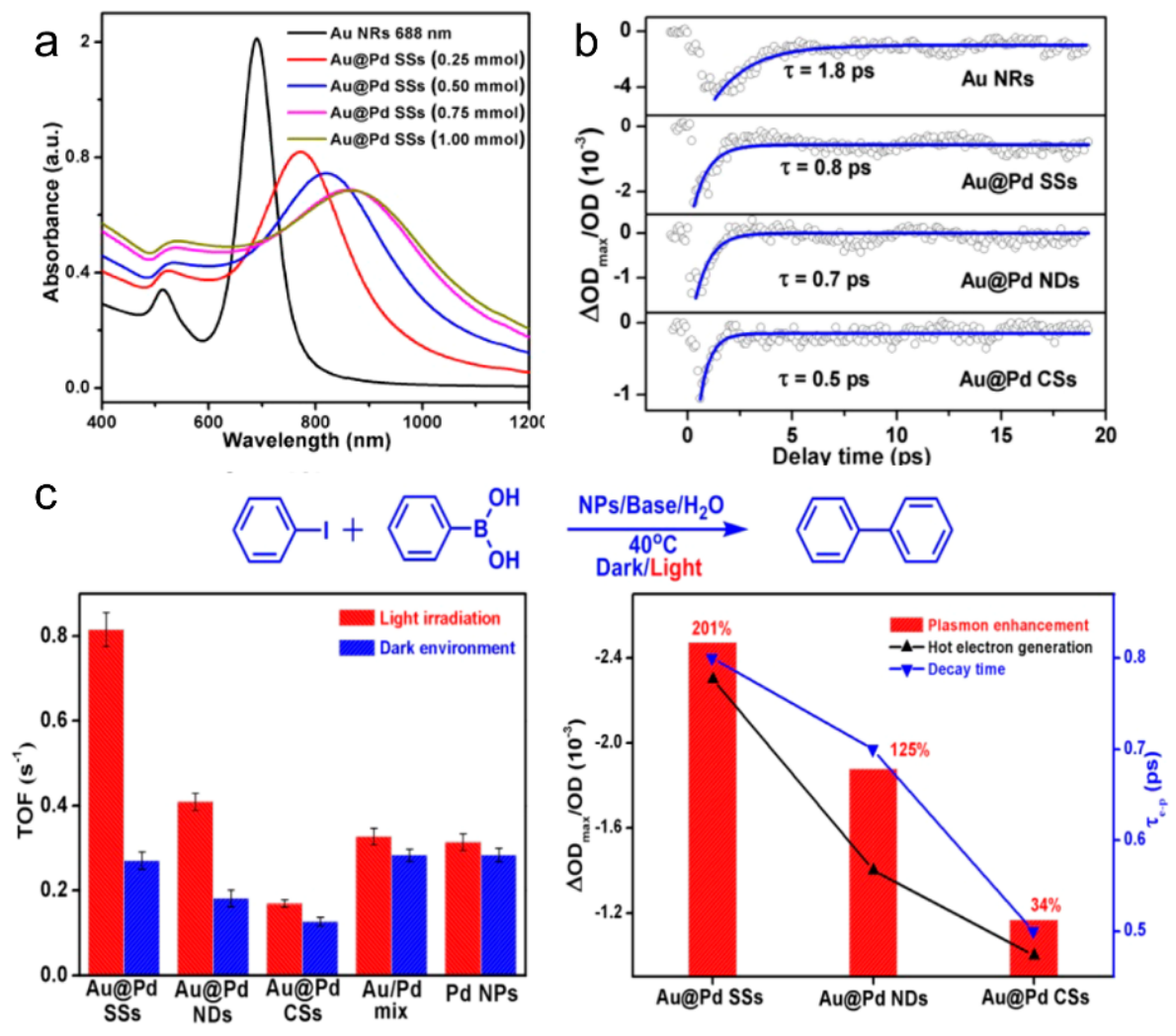

d
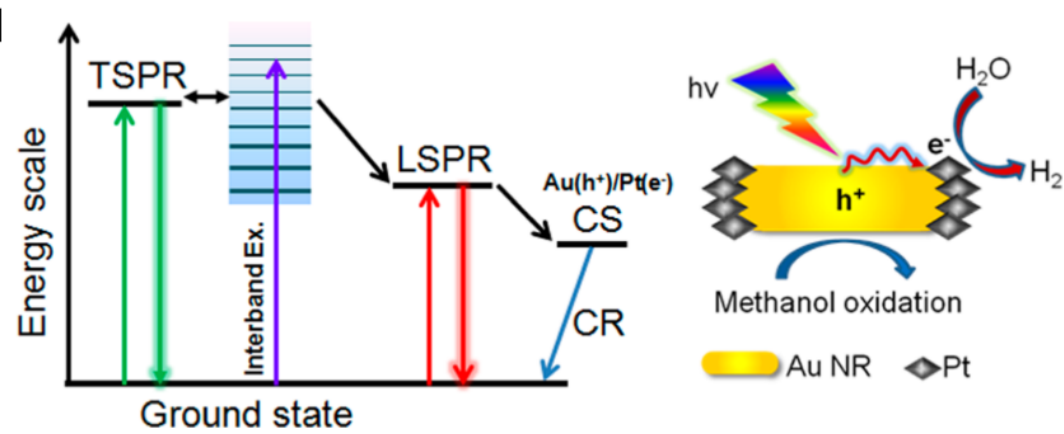

Figure 13. a) LSPR activities of each hybrid NPs. b) The max value (Transient negative change in optical density $\left.\left(\Delta_{\mathrm{OD}}\right)\right)$ divided by its initiate OD against decay time $(\tau)$. c) Plasmon-enhanced Suzuki-Miyaura carbon-carbon coupling reaction with different catalysts. Their catalytic property are evaluated by the TOF, hot electron generation $\left(\Delta O \mathrm{DD}_{\max } / \mathrm{OD}\right)$ and decay time $\left(\tau_{\mathrm{e}-\mathrm{p}}\right)$. Reproduced with permission ${ }^{[54]}$. a-c) Copyright 2017, American Chemical Society. d) Schamatic illustration of $\mathrm{H}_{2}$ evolution from Pt-Au hybrid photocatalysts under laser irradiation. Reproduced with permission ${ }^{[6 a]}$. Copyright 2015, American Chemical Society.

electrons injection into antibonding orbital of surface adsorbed reactants has been increased. For example, the LUMO of iodobenzene were injected with hot electrons, which promoted the Suzuki-Miyaura coupling reaction. Besides, higher efficiency of hot electrons is promoted by the large electromagnetic field on the superstructures. To sum 


\section{WILEY-VCH}

up, turnover frequency (TOF) of Au-Pd superstructures is much higher determined by their superstructure effect (Figure 13c). As for the Pt-tipped Au NRs under visible

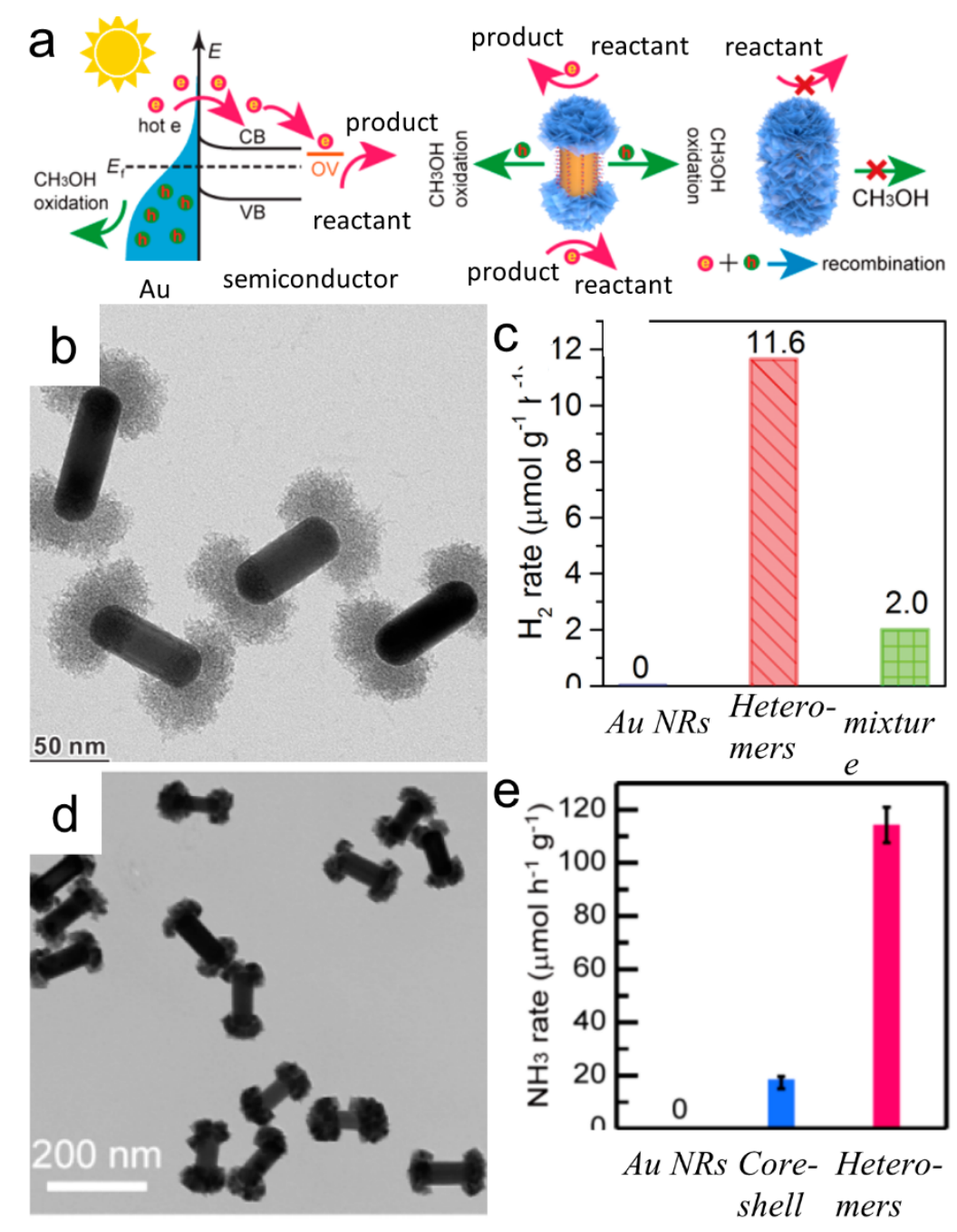

Figure 14. a) Structure effect of plasmonic-semiconductor heterodimeric nanostructures. b) $\mathrm{Au}-\mathrm{TiO}_{2}$ heterodimeric nanostructure and c) its hydrogen evolution reaction rate. d) $\mathrm{Au}-\mathrm{CeO}_{2-\mathrm{x}}$ heterodimeric nanostructure and e) its nitrogen fixation reaction rate. Reproduced with permission ${ }^{[103]}$. a,d,e) Copyright 2019, American Chemical Society. Reproduced with permission ${ }^{[104]}$. b,c) Copyright 2016, American Chemical Society.

light irradiation, hot electrons transfer from $\mathrm{Au}$ surface to Pt. Hydrogen generation evolution reaction occurring on the Pt nanodomains have been promoted by the boosted hot electrons (Figure 13d). After characterization and analysis by the single particle spectroscopies, Pt-tipped Au NRs exhibited higher efficiency of hydrogen generation than recorded on the Pt-covered Au NRs, and uncoated Au NRs, attributed to the higher quenching efficiency of photoluminescence. 


\section{WILEY-VCH}

Plasmonic-semiconductor heteromers also show promising activity for solar energy conversion to fuels via Schottky junction compared to plasmonic-noble metal heteromers. ${ }^{[21]}$ As depicted in Figure 14a, hot electrons generated by the LSPR of Au nanodomains were injected into the $\mathrm{CB}$ of semiconductor
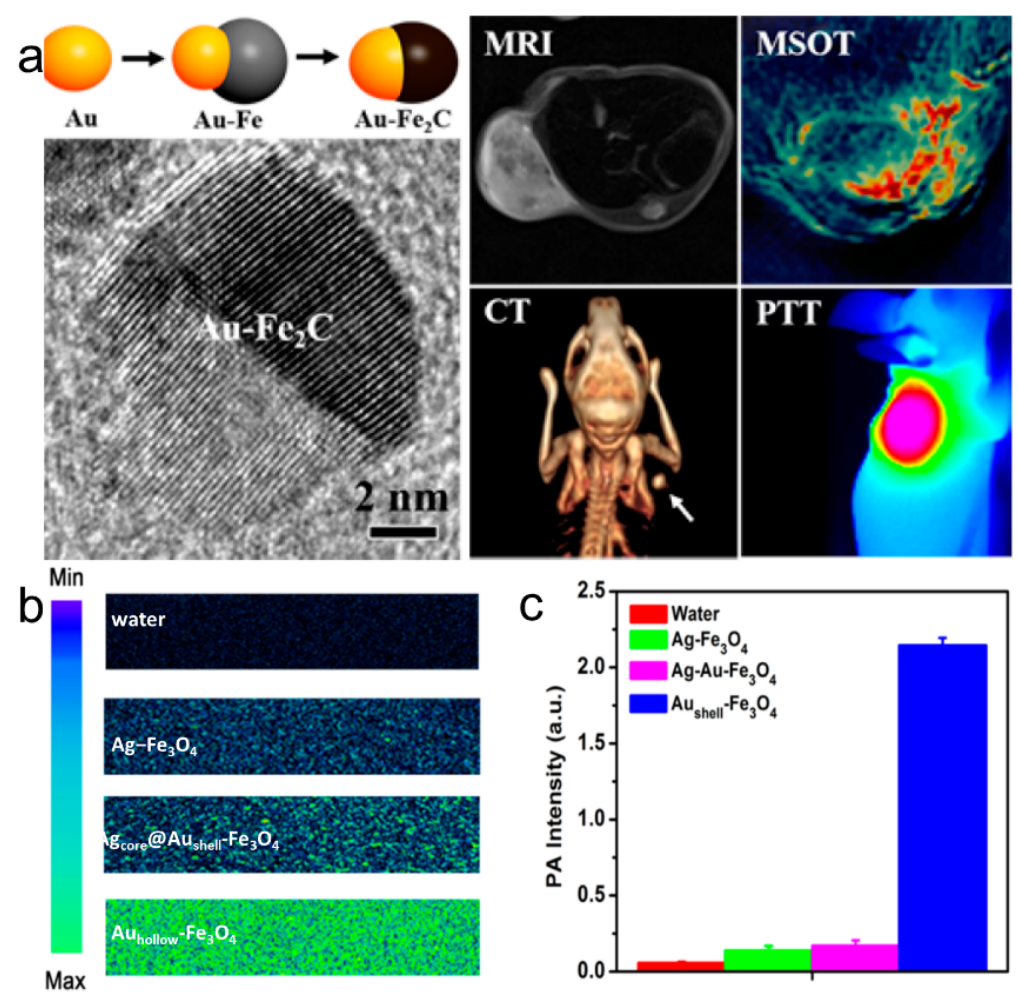

Figure 15. a) $\mathrm{Au}-\mathrm{Fe}_{2} \mathrm{C}$ heteromers are applied for the MRI, CT, MSOT, as well as PTT. Reproduced with permission ${ }^{[9]}$. Copyright 2017, American Chemical Society. b) OCT B-scan images of pure water and aqueous dispersions of $\mathrm{Ag}-\mathrm{Fe}_{3} \mathrm{O}_{4}, \mathrm{Ag}_{\text {core }} @ \mathrm{Au}_{\text {shell- }}$ $\mathrm{Fe}_{3} \mathrm{O}_{4}$, and $\mathrm{Au}_{\text {hollow }}-\mathrm{Fe}_{3} \mathrm{O}_{4}$ heterodimers (from top to bottom). c) PA intensities of aqueous dispersions containing each sample. Reproduced with permission ${ }^{[81]}$. b,c) Copyright 2019, American Chemical Society.

nanodomains. Subsequently, hot charges were transferred into LUMO of reactant molecules, launching the catalytic process. The sacrificed electrons on Au surface need to be supplemented by the electron donors, which can be realized by the plasmonic heteromers rather than core-shell nanostructures. For example, Wu et al. produced $\mathrm{Au}-$ $\mathrm{TiO}_{2}$ nanodumbbells by selectively controlling the concentration of capping agent CTAB on seeds of Au nanorods, as well as the hydrolysis rate of $\mathrm{TiCl}_{3}$ in a suitable $\mathrm{pH}$ 


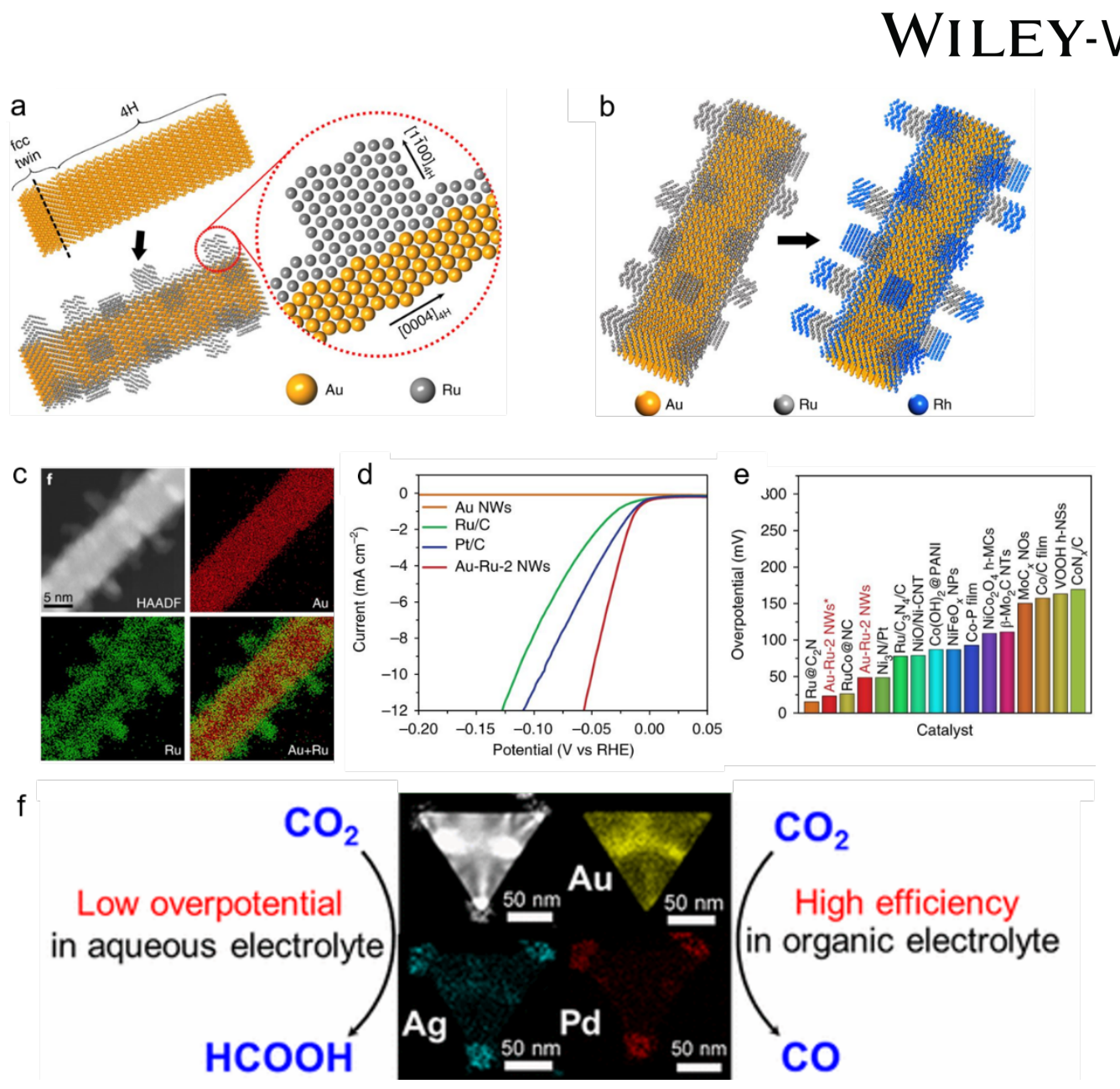

Figure 16. Schematic illustration of the synthesis of a) Au-Ru heterodimeric, b) Au-Ru-Rh ternary nanowires. c) EDX elemental mapping, d) polarization curves and e) overpotential of $\mathrm{Au}-\mathrm{Ru}$ heterodimeric nanowires. Reproduced with permission ${ }^{[105]}$. a-e) Copyright 2018, Nature publishing group. f) AgPd tip-growth on $\mathrm{Au}$ nanoprism for electrocatalytic $\mathrm{CO}_{2}$ reduction. Reproduced with permission ${ }^{[91]}$. Copyright 2017, American Chemical Society.

value. By using a higher concentration of $\mathrm{CTAB}, \mathrm{TiO}_{2}$ selectively deposited on both ends of Au nanorods (Figure 14b). ${ }^{[103]}$ The exposed Au nanorods not only interact with electron donors directly which satisfy the electrons refilling, but also improve the charges separation efficiency by the enhanced electromagnetic field. Besides, $\mathrm{TiO}_{2}$ acts as the electron transfer medium, where hydrogen is produced. As shown at Figure 14c, the dumbbell structures exhibit an excellent plasmon-enhanced hydrogen production under the irradiation of laser compared to other hybrid nanostructures. Those obtained semiconductor domains through hydrolysis of $\mathrm{TiCl}_{3}$ are usually amorphous, which need to undergo higher temperature conditions for crystalline process. Recently, Jia et al. have produced $\mathrm{Au}-\mathrm{CeO}_{2}$ nanodumbells with crystalline ceria phase improving the $\mathrm{N}_{2}$ 


\section{WILEY-VCH}

photoreduction (Figure 14d). ${ }^{[104]}$ The addition of $\mathrm{K}_{2} \mathrm{PtCl}_{4}$ can promote the formation of heteromeric nanostructures by activating the autoredox reaction with the ceria precursor. Few Pt precursors prefer to adsorb on the top of Au nanorods leading to the heteromeric nanostructures, while core-shell nanostructures will be produced if more $\mathrm{K}_{2} \mathrm{PtCl}_{4}$ is deposited on the lateral sides of Au nanorods. The oxygen vacancies on crystalline $\mathrm{CeO}_{2}$ $\mathrm{x}$ are proposed to chemisorb and activate $\mathrm{N}_{2}$ molecules promoting the plasmon-enhanced $\mathrm{N}_{2}$ photofixation under laser illumination. The produced hot electrons on Au nanorods surface transfer into $\mathrm{CB}$ of $\mathrm{CeO}_{2}$, and afterwards they contribute to the reduction of $\mathrm{N}_{2}$ into $\mathrm{NH}_{3}$. Similar to previous results, the structure advantages of heteromers play an important role, which allow it to easily access the electrons donors compared with coreshell nanostructures. So, the photocatalytic efficiency of $\mathrm{N}_{2}$ fixation of heteromers is highest than other hybrid nanostructures (Figure 14e).

Moreover, plasmonic-magnetic heteromers possess superparamagnetic sites and high electron density plasmonic sites, enabling them to be applied in precision diagnosis and tumor therapy. On one hand, their high saturation magnetization $\left(M_{S}\right)$ is due to the reduction of $\mathrm{Fe}^{2+}$ inside $\mathrm{Fe}_{3} \mathrm{O}_{4}$ by the metal adatoms. On the other hand, plasmonic sites with the high electron density can improve the attenuation efficiency for the photothermal therapy (PTT) because of interfacial communication. So, plasmonicmagnetic heteromers, as imaging-guided PTT method, are excellent candidates for the precision diagnosis and cancer therapy. So far, magnetic resonance imaging (MRI), computed tomography (CT), multispectral photoacoustic tomography (MSOT) are still the hybrid imaging modalities, which can provide the size and position information of tumors before and after therapy.[106] Recently, diagnosis with the excellent efficacy with the contrast agents at molecular level are addressed. There is limitation for optimizing the MRI and CT of magnetic-plasmonic core-shell nanostructures because magnetization $\left(\mathrm{M}_{\mathrm{s}}\right)$ is resulted by the magnetic nanodomains. Meanwhile, the LSPR property of plasmonic nanodomains in the plasmonic-magnetic core-shell 


\section{WILEY-VCH}

nanostructures can be blocked by the magnetic shell. Among imaging-guided PTT methods, plasmonic-magnetic heteromers are excellent candidates for the precision of the shielding effect of shell. Plasmonic heteromers allow to efficiently tune their properties and improve the sensitivity of MRI and CT. For example, size tuning of plasmonic or magnetic sites of heteromeric NPs can optimize their X-ray efficiency and transverse relaxivity. ${ }^{76,98}$ As demonstrated in Figure 15a, as-prepared $\mathrm{Au}-\mathrm{Fe}_{2} \mathrm{C}$ heteromers consist of Au with (111) planes and bcc-Fe with (200) planes. Firstly, the size and location of tumors are discerned and in vitro before therapy through MRI, MSOT, and CT imaging. Subsequently, the tumors can be precisely irradiated by laser initiating the PTT effect of plasmonic domains. Finally, the treatment efficiency after therapy can also be precisely evaluated. Tuning the LSPR to the near-infrared region, can not only overcome the water window problem for the laser treatment, but also can match the wavelength of laser leading to largest Optical coherence tomography (OCT) contrast enhancement. Zeng et al. have prepared a series of plasmonic- $\mathrm{Fe}_{3} \mathrm{O}_{4}$ heteromers, the LSPR of which are tuned by the composition control of plasmonic domains. ${ }^{[81]}$ $\mathrm{Au}_{\text {Hollow }}-\mathrm{Fe}_{3} \mathrm{O}_{4}$ heteromers can penetrate longer depth of tissues in vitro leading to the greatest OCT imaging (Figure 15b) and highest photoacoustic signal intensity (Figure 15c). As a proof of concept, plasmonic-magnetic heteromeric NPs are excellent contrast agents for those multi-modality bioimaging. MRI signal intensity decreased a lot and the $\mathrm{CT}$ values somewhat increased after the injection of $\mathrm{Au}-\mathrm{Fe}_{3} \mathrm{O}_{4}$ into rats body.

Apart from the LSPR effect, such kind of plasmonic heteromers are good candidates to maximize the electrocatalytic activity by selectively choosing the reaction pathways, and d-states of metal nanodomains at the Fermi level. As a result, plasmonic heteromers as electrocatalysts will increase the turnover number (TON) or turnover frequency (TOF), selectivity, efficiency and stability. For example, the adsorbed energy of reactants strongly relies on the surface electronic structure of transition metal nanocatalysts. ${ }^{[107]}$ Kim et al. have tuned the electronic effect determined by position and 


\section{WILEY-VCH}

width of d-band of metal nanodomains in order to control the energetics of intermediate binding strength towards to $\mathrm{CO}_{2} \mathrm{RR}$ enhancing the catalytic activity. ${ }^{[108]}$ Unconventional phase and phase transformation of nanomaterials also can govern the controlled synthesis of heteronanostructures, besides the size, morphology, compositions, facets and crystallinity of seeds. ${ }^{[109]}$ Normally, there are ordered or periodic atomic arrangements in the phase of nanocrystals. Zhang and co-workers have developed a series of plasmonic heteromers using the seeds with an unusual crystal phase. The unconvential phase of seeds, which have improved electrocatalytic property.[110] Recently, $\mathrm{Ru}$ nanorods preferred to vertically epitaxial grow on $4 \mathrm{H} \mathrm{Au}$ nanowires compared to fcc Au nanowires (Figure 16a,b), because of closed hexagonal 4H phase with priority epitaxial growth sites and large lattice mismatch. Their HAADF images and EDX mapping are shown in the Figure 16c. In this case, one-dimensional structure decreases the charge transfer resistance. Furthermore, growth of Ru nanorods on $\mathrm{Au}$ nanowires optimizing the electronic band structure, lead to $\mathrm{Au}-\mathrm{Ru}$ hybrid nanowires with excellent hydrogen evolution reaction (HER) performance in alkaline media, compared to $4 \mathrm{H} \mathrm{Au}$ nanowires which showed no activity (Figure 16d). The lower Tafel slope of $\mathrm{Au}-\mathrm{Ru}$ hybrid nanowires revealed that HER reaction is more preferable on their surface compared to that of $\mathrm{Ru} / \mathrm{C}$ catalysts. Furthermore, turnover frequency of $\mathrm{Au}-\mathrm{Ru}$ hybrid nanowires is higher than $\mathrm{Ru} / \mathrm{C}$ or $\mathrm{Pt} / \mathrm{C}$ catalysts (Figure 16e). Seed-mediated approaches have also been extended to prepare ternary heteromers $\mathrm{Au}-\mathrm{Ru}-\mathrm{X}(\mathrm{X}=\mathrm{Rh}, \mathrm{Pt})$ when $\mathrm{Au}-$ $\mathrm{Ru}$ hybrids were applied as seeds (Figure 16b). Shan et al. have selectively deposited multibranched AgPd nanodendrites at the surface of Au prisms through adjusting the reduction rate of metal adatoms. ${ }^{97}$ Electrons at high curvature sites of Au nanoprisms possess particularly high reactivity, allowing to initiate galvanic replacement reaction of Ag shell with $\mathrm{H}_{2} \mathrm{PdCl}_{4}$. When the deposition rate is lower than the diffusion rate, a lower amount of electrons is produced leading to AgPd nanodendrites edged-growth on $\mathrm{Au}$ nanoprisms. In contrast, overabundance of electrons can be accumulated on the seeds 


\section{WILEY-VCH}

surface leading to the AgPd nanodendrites tip-growth Au nanoprisms. Importantly, the ratio between the deposition rate and the diffusion rate can be controlled by tuning the ratio between $\mathrm{Ag}$ and $\mathrm{Pd}$ precursors. As seen in Figure 16f, AgPd nanodendritesmodified $\mathrm{Au}$ metal NPs provided more active edge and corner sites for the $\mathrm{CO}_{2} \mathrm{RR}$ compared to AgPd nanodendrites. The short distance between the Ag and Pd for surface stabilization of $\mathrm{H}^{+}$and $\mathrm{CO}_{2}$ can promote the formation of $\mathrm{C}-\mathrm{H}$ bonds for formate production. Besides, Au cores not only provide chemical stability to support AgPd nanodendrites on their surface but also exhibit high electrical conductivity to promote fast electron transfer. ${ }^{97}$

\section{Conclusion and perspective}

Plasmonic heteromers are composed of different metal inorganic functional nanodomains and at least one nanodomain is plasmonic metallic nanodomains. In this review, we have summarized the recent advances on the synthesis of plasmonic heteromers which are fused with noble metal, magnetic, semiconductor domains via the solid-solid nanointerface. Among the various approaches, seed-mediated approach is one efficient route to selectively engineer the secondary metal adatoms on the desired sites of seeds via competing against the homogeneous nucleation and growth. The thermodynamics and kinetics parameters to accurately control the morphology and composition of plasmonic heteromeric nanostructures were addressed in detail. The review covers the simultaneous effect of thermodynamics and kinetic control for tuning the synthesis and application of plasmonic metallic heteromers.

Thanks to the structure advantages of plasmonic heteromeric nanostructures, their performance in real-world applications has been extended. Strengths of plasmonic-noble metal, plasmonic-semiconductor, plasmonic-magnetic heteromers are highlighted, along with the most recent developments in applications in the fields of photocatalysis, electrocatalysis, bioimaging and cancer therapy. Although there have been numerous successful cases to construct the plasmonic heteromers with structure and composition 


\section{WILEY-VCH}

diversity, there are still several key challenges for their synthesis and enhanced properties. Firstly, considering that the performance strongly depends on the morphologies and compositions, there is still a big challenge to construct uniform and versatile morphology of heteromeric nanostructures composed by more than two functional nanodomains. For example, shape-directing of the secondary metallic nanodomains grown on seeds is still hard to obtain because the growth modes depend on the kinetic (injection rate of adatoms) and thermodynamics parameters (e.g., quality of seeds, capping agents, $\mathrm{pH}$ value, temperature, among others). Secondly, the deposition of secondary metallic adatoms on the surface of seeds is rather too fast to be in-situ monitored, and this complicates the full understanding of the growth mechanisms. It is often not so clear if metal adatoms are deposited on the sides of seeds in the beginning, and then diffused to the tips of seeds for the creation of heteromers, or if they are directly deposited at the tips of seeds. ${ }^{[111]}$ Therefore, high resolution technologies should be employed and further developped for the in-situ characterization of heterogeneous growth process. Finally, plasmonic metallic heteromers have already been used in several applications, however, there are still challenging goals, such as the improvement of energy conversion efficiency from green energy (solar and electricity energy) to chemical energy through the nanostructure design of plasmonic metallic heteromers. Further systematic research both in experiment and theory is still needed for gaining more fundamental understanding and enhancing the range of potential applications of plasmonic heteromers. Future research should probably take carefully into account the crucial parameters, thermodynamics and kinetics, which affect the plasmonic heteromer growth, in order to design novel materials of this family, with finely tuned properties, being suitable for a variety of applications. We strongly believe that this review will help the research community which works in this topic to quickly find a comprehensive guide containing the key points for the optimum structural design of plasmonic heteromers. 


\section{Conflicts of interest}

The authors declare no conflict of interest.

\section{Acknowledgements}

This work was financially supported by Natural Science Foundation of China (NO. 21902148).

\section{References}

[1] P. Herves, M. Perez-Lorenzo, L. M. Liz-Marzan, J. Dzubiella, Y. Lu, M. Ballauff, Chem. Soc. Rev. 2012, 41, 5577.

[2] S. Gomez-Grana, B. Goris, T. Altantzis, C. Fernandez-Lopez, E. Carbo-Argibay, A. GuerreroMartinez, N. Almora-Barrios, N. Lopez, I. Pastoriza-Santos, J. Perez-Juste, S. Bals, G. Van Tendeloo, L. M. Liz-Marzan, J. Phys. Chem. Lett. 2013, 4, 2209.

[3] Y. Wu, D. S. Wang, X. B. Chen, G. Zhou, R. Yu, Y. D. Li, J. Am. Chem. Soc. 2013, 135, 12220.

[4] a) C. Chen, Y. J. Kang, Z. Y. Huo, Z. W. Zhu, W. Y. Huang, H. L. L. Xin, J. D. Snyder, D. G. Li, J. A. Herron, M. Mavrikakis, M. F. Chi, K. L. More, Y. D. Li, N. M. Markovic, G. A. Somorjai, P. D. Yang, V. R. Stamenkovic, Science 2014, 343, 1339; b) M. A. Mahmoud, W. Qian, M. A. El-Sayed, Nano Lett 2011, 11, 3285; c) L. Polavarapu, L. M. Liz-Marzan, Nanoscale 2013, 5, 4355; d) L. Polavarapu, D. Zanaga, T. Altantzis, S. Rodal-Cedeira, I. Pastoriza-Santos, J. PerezJuste, S. Bals, L. M. Liz-Marzan, J. Am. Chem. Soc. 2016, 138, 11453.

[5] a) C. Langhammer, Z. Yuan, I. Zoric, B. Kasemo, Nano Lett. 2006, 6, 833; b) X. H. Li, J. Lian, M. Lin, Y. T. Chan, J. Am. Chem. Soc. 2011, 133, 672; c) K. F. Wu, W. E. Rodriguez-Cordoba, Y. Yang, T. Q. Lian, Nano Lett. 2013, 13, 5255.

[6] a) Z. K. Zheng, T. Tachikawa, T. Majima, J. Am. Chem. Soc. 2014, 136, 6870; b) M. Grzelczak, J. Perez-Juste, B. Rodriguez-Gonzalez, L. M. Liz-Marzan, J. Mater. Chem. 2006, 16, 3946; c) S. M. Kim, S. J. Lee, S. H. Kim, S. Kwon, K. J. Yee, H. Song, G. A. Somorjai, J. Y. Park, Nano Lett. 2013, 13, 1352.

[7] R. F. Dong, Q. L. Zhang, W. Gao, A. Pei, B. Y. Ren, Acs Nano 2016, 10, 839.

[8] a) Y. Y. Tian, Z. H. Shuai, J. J. Shen, L. Zhang, S. F. Chen, C. Y. Song, B. M. Zhao, Q. L. Fan, L. H. Wang, Small 2018, 14, 1800669; b) X. Chen, M. M. Liang, J. Xu, H. L. Sun, C. Wang, J. Wei, H. Zhang, W. M. Yang, Z. L. Yang, J. J. Sun, Z. Q. Tian, J. F. Li, Nanoscale 2020, 12, 5341.

[9] Y. M. Ju, H. L. Zhang, J. Yu, S. Y. Tong, N. Tian, Z. Y. Wang, X. B. Wang, X. T. Su, X. Chu, J. Lin, Y. Ding, G. J. Li, F. G. Sheng, Y. L. Hou, Acs Nano 2017, 11, 9239.

[10] a) G. Zheng, E. Carbo-Argibay, E. Gonzalez-Romero, I. Pastoriza-Santos, J. Perez-Juste, Cryst. Growth. Des. 2020, DOI: 10.1021/acs.cgd.0c00500; b) Z. Y. Wu, L. Li, T. Liao, X. Q. Chen, W. Jiang, W. Luo, J. P. Yang, Z. Q. Sun, Nano Today 2018, 22, 62.

[11] G. C. Zheng, Z. Y. Rao, J. Perez-Juste, R. L. Du, W. Liu, J. Y. Dai, W. Zhang, L. Y. S. Lee, K. Y. Wong, Angew. Chem. Int. Edit. 2018, 57, 16452.

[12] a) J. C. Qiu, M. H. Xie, Z. H. Lyu, K. D. Gilroy, H. Liu, Y. N. Xia, Nano Lett. 2019, 19, 6703; b) F. Wang, S. Cheng, Z. H. Bao, J. F. Wang, Angew. Chem. Int. Edit. 2013, 52, 10344.

[13] a) Y. Pan, J. H. Gao, B. Zhang, X. X. Zhang, B. Xu, Langmuir 2010, 26, 4184; b) J. H. Yoon, Y. Zhou, M. G. Blaber, G. C. Schatz, S. Yoon, J. Phys. Chem. Lett. 2013, 4, 1371.

[14] a) S. J. Guo, J. Li, S. J. Dong, E. K. Wang, J. Phys. Chem. C. 2010, 114, 15337; b) H. J. Wang, S. L. Yin, Y. Xu, X. N. A. Li, A. A. Alshehri, Y. Yamauchi, H. R. Xue, Y. V. Kaneti, L. Wang, J. Mater. Chem. A 2018, 6, 8662.

[15] a) R. F. Shepherd, J. C. Conrad, S. K. Rhodes, D. R. Link, M. Marquez, D. A. Weitz, J. A. Lewis, Langmuir 2006, 22, 8618; b) V. Sebastian, N. Zaborenko, L. Gu, K. F. Jensen, Cryst. Growth. Des. 2017, 17, 2700.

[16] N. R. Jana, L. Gearheart, C. J. Murphy, Adv. Mater., 2001, 13, 1389.

[17] a) D. D. Li, J. Wang, G. C. Zheng, J. H. Liu, W. H. Xu, Nanotechnology 2013, 24, 487; b) J. Y. Xu, J. Wang, L. T. Kong, G. C. Zheng, Z. Guo, J. H. Liu, J. Raman. Spectrosc. 2011, 42, 1728; c) M. Samim, C. K. Prashant, A. K. Dinda, A. N. Maitra, I. Arora, Int. J. Nanomed. 2011, 6, 1825; d) M. Grzelczak, J. Perez-Juste, P. Mulvaney, L. M. Liz-Marzan, Chem. Soc. Rev. 2008, 37, 1783.

[18] Y. M. Lee, M. A. Garcia, N. A. F. Huls, S. H. Sun, Angew. Chem. Int. Edit. 2010, 49, 1271.

[19] a) I. Pastoriza-Santos, C. Kinnear, J. Perez-Juste, P. Mulvaney, L. M. Liz-Marzan, Nat. Rev. Mater. 2018, 3, 375; b) A. U. Khan, Y. C. Guo, X. Chen, G. L. Liu, Acs Nano 2019, 13, 4255.

[20] Z. K. Zheng, T. Tachikawa, T. Majima, J. Am. Chem. Soc. 2015, 137, 948. 
[21] Z. W. Seh, S. H. Liu, M. Low, S. Y. Zhang, Z. L. Liu, A. Mlayah, M. Y. Han, Adv. Mater. 2012 , 24, 2310.

[22] a) Z. C. Zhang, B. Xu, X. Wang, Chem. Soc. Rev. 2014, 43, 7870; b) B. H. Wu, N. F. Zheng, Nano Today 2013, 8, 168; c) H. L. Jiang, Q. Xu, J. Mater. Chem. 2011, 21, 13705; d) Z. C. Zhang, Y. Liu, B. Chen, Y. Gong, L. Gu, Z. X. Fan, N. L. Yang, Z. C. Lai, Y. Chen, J. Wang, Y. Huang, M. Sindoro, W. X. Niu, B. Li, Y. Zong, Y. H. Yang, X. Huang, F. W. Huo, W. Huang, H. Zhang, Adv. Mater. 2016, 28, 10282.

[23] Y. M. Tan, J. M. Fan, G. X. Chen, N. F. Zheng, Q. J. Xie, Chem. Commun. 2011, 47, 11624.

[24] Y. E. Wu, D. S. Wang, G. Zhou, R. Yu, C. Chen, Y. D. Li, J. Am. Chem. Soc. 2014, 136, 11594.

[25] T. R. Gordon, R. E. Schaak, Chem. Mater. 2014, 26, 5900.

[26] a) C. J. DeSantis, R. G. Weiner, A. Radmilovic, M. M. Bower, S. E. Skrabalak, J. Phys. Chem. Lett. 2013, 4, 3072; b) L. Carbone, P. D. Cozzoli, Nano Today 2010, 5, 449; c) N. E. Motl, A. F. Smith, C. J. DeSantis, S. E. Skrabalak, Chem. Soc. Rev. 2014, 43, 3823; d) M. R. Jones, K. D. Osberg, R. J. Macfarlane, M. R. Langille, C. A. Mirkin, Chem. Rev. 2011, 111, 3736; e) C. B. Gao, J. Goebl, Y. D. Yin, J. Mater. Chem. C 2013, 1, 3898.

[27] a) Y. N. Xia, X. H. Xia, H. C. Peng, J. Am. Chem. Soc. 2015, 137, 7947; b) X. M. Lu, M. Rycenga, S. E. Skrabalak, B. Wiley, Y. N. Xia, Annu. Rev. Phys. Chem. 2009, 60, 167; c) K. D. Gilroy, A. Ruditskiy, H. C. Peng, D. Qin, Y. N. Xia, Chem. Rev. 2016, 116, 10414; d) Y. N. Xia, Y. J. Xiong, B. Lim, S. E. Skrabalak, Angew. Chem. Int. Edit. 2009, 48, 60; e) Y. N. Xia, K. D. Gilroy, H. C. Peng, X. H. Xia, Angew. Chem. Int. Edit. 2017, 56, 60.

[28] a) R. Costi, A. E. Saunders, U. Banin, Angew. Chem. Int. Edit. 2010, 49, 4878; b) U. Banin, Y. Ben-Shahar, K. Vinokurov, Chem. Mater.2014, 26, 97; c) P. D. Cozzoli, T. Pellegrino, L. Manna, Chem. Soc. Rev. 2006, 35, 1195; d) M. R. Buck, R. E. Schaak, Angew. Chem. Int. Edit. 2013, 52, 6154; e) H. Y. Zheng, Y. J. Li, H. B. A. Liu, X. D. Yin, Y. L. Li, Chem Soc Rev 2011, 40, 4506; f) Y. G. Sun, Natl. Sci. Rev. 2015, 2, 329; g) J. Hu, S. X. Zhou, Y. Y. Sun, X. S. Fang, L. M. Wu, Chem. Soc. Rev. 2012, 41, 4356.

[29] G. C. Zheng, J. Wang, L. T. Kong, H. F. Cheng, J. H. Liu, Plasmonics 2012, 7, 487.

[30] X. Q. Huang, Y. J. Li, H. L. Zhou, X. Zhong, X. F. Duan, Y. Huang, Chem-Eur. J. 2012, 18, 9505 .

[31] M. Kluenker, B. M. Connolly, D. M. Marolf, M. N. Tahir, K. Korschelt, P. Simon, U. Kohler, S. Plana-Ruiz, B. Barton, M. Panthofer, U. Kolb, W. Tremel, Inorg. Chem. 2018, 57, 13640.

[32] Y. U. Xiang, X. C. Wu, D. F. Liu, Z. Y. Li, W. G. Chu, L. L. Feng, K. Zhang, W. Y. Zhou, S. S. Xie, Langmuir 2008, 24, 3465.

[33] W. Q. Zhang, J. Liu, W. X. Niu, H. Yan, X. M. Lu, B. Liu, Acs. Appl. Mater. Inter. 2018, 10, 14850 .

[34] M. Mayer, L. Scarabelli, K. March, T. Altantzis, M. Tebbe, M. Kociak, S. Bals, F. J. G. de Abajo, A. Fery, L. M. Liz-Marzan, Nano Lett. 2015, 15, 5427.

[35] M. Luo, A. Ruditskiy, H. C. Peng, J. Tao, L. Figueroa-Cosme, Z. K. He, Y. N. Xia, Adv. Funct. Mater. 2016, 26, 1209.

[36] a) D. J. Milliron, S. M. Hughes, Y. Cui, L. Manna, J. B. Li, L. W. Wang, A. P. Alivisatos, Nature 2004, 430, 190; b) J. Yang, H. I. Elim, Q. B. Zhang, J. Y. Lee, W. Ji, J. Am. Chem. Soc. 2006, 128, 11921; c) L. Wang, Y. Nemoto, Y. Yamauchi, J. Am. Chem. Soc. 2011, 133, 9674; d) B. Lim, M. J. Jiang, P. H. C. Camargo, E. C. Cho, J. Tao, X. M. Lu, Y. M. Zhu, Y. N. Xia, Science 2009, 324, 1302.

[37] a) M. R. Langille, J. Zhang, C. A. Mirkin, Angew. Chem. Int. Edit. 2011, 50, 3543; b) A. SanchezIglesias, N. Winckelmans, T. Altantzis, S. Bals, M. Grzelczak, L. M. Liz-Marzan, J. Am. Chem. Soc. 2017, 139, 107; c) G. Gonzalez-Rubio, T. M. de Oliveira, T. Altantzis, A. La Porta, A. Guerrero-Martinez, S. Bals, L. Scarabelli, L. M. Liz-Marzan, Chem. Commun. 2017, 53, 11360.

[38] Y. Chen, Z. C. Lai, X. Zhang, Z. X. Fan, Q. Y. He, C. L. Tan, H. Zhang, Nat. Rev. Chem. 2020, 4, 243.

[39] J. Liu, J. T. Zhang, Chem. Rev. 2020, 120, 2123.

[40] T. Bala, A. Sanyal, A. Singh, D. Kelly, C. O'Sullivan, F. Laffir, K. M. Ryan, J. Mater. Chem. 2011, 21, 6815 .

[41] D. S. Li, M. H. Nielsen, J. R. I. Lee, C. Frandsen, J. F. Banfield, J. J. De Yoreo, Science 2012, $336,1014$.

[42] Z. M. Peng, H. Yang, Nano Today 2009, 4, 143.

[43] C. Zhu, J. Zeng, J. Tao, M. C. Johnson, I. Schmidt-Krey, L. Blubaugh, Y. M. Zhu, Z. Z. Gu, Y. N. Xia, J. Am. Chem. Soc. 2012, 134, 15822.

[44] B. Lim, H. Kobayashi, T. Yu, J. G. Wang, M. J. Kim, Z. Y. Li, M. Rycenga, Y. N. Xia, J. Am. Chem. Soc. 2010, 132, 2506.

[45] X. H. Xia, S. F. Xie, M. C. Liu, H. C. Peng, N. Lu, J. G. Wang, M. J. Kim, Y. N. Xia, P. Natl. Acad. Sci. USA 2013, 110, 6669. 
[46] J. Zeng, C. Zhu, J. Tao, M. S. Jin, H. Zhang, Z. Y. Li, Y. M. Zhu, Y. N. Xia, Angew. Chem. Int. Edit. 2012, 51, 2354.

[47] a) Z. Y. Bao, D. Y. Lei, R. B. Jiang, X. Liu, J. Y. Dai, J. F. Wang, H. L. W. Chan, Y. H. Tsang, Nanoscale 2014, 6, 9063; b) Z. M. Peng, H. Yang, J. Am. Chem. Soc. 2009, 131, 7542; c) P. J. Straney, L. E. Marbella, C. M. Andolina, N. T. Nuhfer, J. E. Millstone, J. Am. Chem. Soc. 2014, 136, 7873; d) S. J. Guo, S. J. Dong, E. K. Wang, Acs Nano 2010, 4, 547; e) Z. Z. Lou, M. Fujitsuka, T. Majima, Acs Nano 2016, 10, 6299.

[48] F. R. Fan, D. Y. Liu, Y. F. Wu, S. Duan, Z. X. Xie, Z. Y. Jiang, Z. Q. Tian, J. Am. Chem. Soc. 2008, 130, 6949 .

[49] M. Grzelczak, J. Perez-Juste, F. J. G. de Abajo, L. M. Liz-Marzan, J. Phys. Chem. C 2007, 111, 6183.

[50] G. C. Zheng, S. de Marchi, V. Lopez-Puente, K. Sentosun, L. Polavarapu, I. Perez-Juste, E. H. Hill, S. Bals, L. M. Liz-Marzan, I. Pastoriza-Santos, J. Perez-Juste, Small 2016, 12, 3935.

[51] a) X. Q. Huang, S. H. Tang, X. L. Mu, Y. Dai, G. X. Chen, Z. Y. Zhou, F. X. Ruan, Z. L. Yang, N. F. Zheng, Nat. Nanotechnol. 2011, 6, 28; b) L. Scarabelli, M. Coronado-Puchau, J. J. GinerCasares, J. Langer, L. M. Liz-Marzan, Acs Nano 2014, 8, 5833; c) C. L. Johnson, E. Snoeck, M. Ezcurdia, B. Rodriguez-Gonzalez, I. Pastoriza-Santos, L. M. Liz-Marzan, M. J. Hytch, Nat. Mater. 2008, 7, 120; d) S. Y. Liu, W. X. Niu, S. Firdoz, W. Q. Zhang, Langmuir 2017, 33, 12254.

[52] a) S. Mourdikoudis, M. Chirea, D. Zanaga, T. Altantzis, M. Mitrakas, S. Bals, L. M. Liz-Marzan, J. Perez-Juste, I. Pastoriza-Santos, Nanoscale 2015, 7, 8739; b) S. Mourdikoudis, T. Altantzis, L. M. Liz-Marzan, S. Bals, I. Pastoriza-Santos, J. Perez-Juste, Crystengcomm. 2016, 18, 3422.

[53] S. E. Lohse, N. D. Burrows, L. Scarabelli, L. M. Liz-Marzan, C. J. Murphy, Chem. Mater. 2014, $26,34$.

[54] J. Guo, Y. Zhang, L. Shi, Y. F. Zhu, M. F. Mideksa, K. Hou, W. S. Zhao, D. W. Wang, M. T. Zhao, X. F. Zhang, J. W. Lv, J. Q. Zhang, X. L. Wang, Z. Y. Tang, J. Am. Chem. Soc. 2017, 139, 17964.

[55] J. H. Zhu, J. J. Wu, F. Liu, R. J. Xing, C. Z. Zhang, C. Yang, H. Yin, Y. L. Hou, Nanoscale 2013 , 5,9141 .

[56] H. Schlicke, D. Ghosh, L. K. Fong, H. L. L. Xin, H. M. Zheng, A. P. Alivisatos, Angew. Chem. Int. Edit. 2013, 52, 980.

[57] a) J. M. Li, J. Y. Liu, Y. Yang, D. Qin, J. Am. Chem. Soc. 2015, 137, 7039; b) D. Qin, Y. Zhang, Y. R. Wu, Abstr. Pap. Am. Chem. S. 2017, 254; c) J. M. Li, X. J. Sun, D. Qin, Chemnanomat. 2016, 2, 494; d) Y. C. Qin, W. L. Zhang, K. Guo, X. B. Liu, J. Q. Liu, X. Y. Liang, X. P. Wang, D. W. Gao, L. Y. Gan, Y. T. Zhu, Z. C. Zhang, W. P. Hu, Adv. Funct. Mater. 2020, 30.

[58] a) X. H. Xia, Y. Wang, A. Ruditskiy, Y. N. Xia, Adv. Mater. 2013, 25, 6313; b) H. Zhang, M. S. Jin, J. G. Wang, W. Y. Li, P. H. C. Camargo, M. J. Kim, D. R. Yang, Z. X. Xie, Y. A. Xia, J. Am. Chem. Soc. 2011, 133, 6078.

[59] E. Gonzalez, J. Arbiol, V. F. Puntes, Science 2011, 334, 1377.

[60] Z. M. Tang, B. C. Ye, S. S. Han, T. J. Lee, S. H. Bhang, W. S. Kim, T. Yu, Nano Converg. 2019, 6,38

[61] W. Q. Zhang, J. Z. Yang, X. M. Lu, Acs Nano 2012, 6, 7397.

[62] P. S. Lutz, I. T. Bae, M. M. Maye, Nanoscale 2015, 7, 15748.

[63] Y. Yu, Q. B. Zhang, Q. F. Yao, J. P. Xie, J. Y. Lee, Chem. Mater. 2013, 25, 4746.

[64] X. Guo, Q. Zhang, Y. H. Sun, Q. Zhao, J. Yang, Acs Nano 2012, 6, 1165.

[65] a) C. L. Xiao, H. Hu, X. Y. Zhang, D. R. MacFarlane, Acs. Sustain. Chem. Eng. 2018, 6, 2806; b) A. Figuerola, M. van Huis, M. Zanella, A. Genovese, S. Marras, A. Falqui, H. W. Zandbergen, R. Cingolani, L. Manna, Nano Lett. 2010, 10, 3028.

[66] Y. Choi, G. H. Ryu, S. H. Min, B. R. Lee, M. H. Song, Z. Lee, B. S. Kim, Acs Nano 2014, 8, 11377.

[67] P. Li, Z. Wei, T. Wu, Q. Peng, Y. D. Li, J. Am. Chem. Soc. 2011, 133, 5660.

[68] R. Costi, A. E. Saunders, E. Elmalem, A. Salant, U. Banin, Nano Lett. 2008, 8, 637.

[69] Z. W. Seh, S. H. Liu, S. Y. Zhang, M. S. Bharathi, H. Ramanarayan, M. Low, K. W. Shah, Y. W. Zhang, M. Y. Han, Angew. Chem. Int. Edit. 2011, 50, 10140.

[70] L. Weng, H. Zhang, A. O. Govorov, M. Ouyang, Nat Commun 2014, 5, 4792.

[71] Y. R. Wu, X. J. Sun, Y. Yang, J. M. Li, Y. Zhang, D. Qin, Accounts. Chem. Res. 2017, 50, 1774.

[72] J. T. Zhang, Y. Tang, K. Lee, O. Y. Min, Science 2010, 327, 1634.

[73] F. H. Lin, R. A. Doong, J. Phys. Chem. C 2011, 115, 6591.

[74] S. Liang, X. L. Liu, Y. Z. Yang, Y. L. Wang, J. H. Wang, Z. J. Yang, L. B. Wang, S. F. Jia, X. F. Yu, L. Zhou, J. B. Wang, J. Zeng, Q. Q. Wang, Z. Y. Zhang, Nano Lett. 2012, 12, 5281.

[75] a) R. Scarfiello, C. Nobile, P. D. Cozzoli, Front. Mater. 2016, 3, 56; b) C. Xu, J. Xie, D. Ho, C. Wang, N. Kohler, E. G. Walsh, J. R. Morgan, Y. E. Chin, S. Sun, Angew. Chem. Int. Edit. 2008, 47,173 . 
[76] a) H. Yu, M. Chen, P. M. Rice, S. X. Wang, R. L. White, S. H. Sun, Nano Lett. 2005, 5, 379; b) W. L. Shi, H. Zeng, Y. Sahoo, T. Y. Ohulchanskyy, Y. Ding, Z. L. Wang, M. Swihart, P. N. Prasad, Nano Lett. 2006, 6, 875.

[77] P. Tancredi, L. S. da Costa, S. Calderon, O. Moscoso-Londono, L. M. Socolovsky, P. J. Ferreira, D. Muraca, D. Zanchet, M. Knobel, Nano Res. 2019, 12, 1781.

[78] G. M. Jiang, Y. X. Huang, S. Zhang, H. Y. Zhu, Z. B. Wu, S. H. Sun, Nanoscale 2016, 8, 17947.

[79] F. H. Lin, W. Chen, Y. H. Liao, R. A. Doong, Y. D. Li, Nano Res. 2011, 4, 1223.

[80] S. Peng, C. H. Lei, Y. Ren, R. E. Cook, Y. G. Sun, Angew. Chem. Int. Edit. 2011, $50,3158$.

[81] J. B. Zeng, M. F. Gong, D. W. Wang, M. M. Li, W. J. Xu, Z. W. Li, S. C. Li, D. Zhang, Z. F. Yan, Y. D. Yin, Nano Lett. 2019, 19, 3011.

[82] M. Grzelczak, J. Perez-Juste, B. Rodriguez-Gonzalez, M. Spasova, I. Barsukov, M. Farle, L. M. Liz-Marzan, Chem. Mater. 2008, 20, 5399.

[83] J. S. Choi, Y. W. Jun, S. I. Yeon, H. C. Kim, J. S. Shin, J. Cheon, J. Am. Chem. Soc. 2006, 128, 15982.

[84] X. C. Ye, D. R. Hickey, J. Y. Fei, B. T. Diroll, T. Paik, J. Chen, C. B. Murray, J. Am. Chem. Soc. 2014, 136, 5106.

[85] J. K. Norskov, T. Bligaard, A. Logadottir, S. Bahn, L. B. Hansen, M. Bollinger, H. Bengaard, B. Hammer, Z. Sljivancanin, M. Mavrikakis, Y. Xu, S. Dahl, C. J. H. Jacobsen, J. Catal. 2002, 209, 275.

[86] M. R. Buck, J. F. Bondi, R. E. Schaak, Nat. Chem. 2012, 4, 37.

[87] I. Schick, D. Gehrig, M. Montigny, B. Balke, M. Panthofer, A. Henkel, F. Laquai, W. Tremel, Chem. Mater. 2015, 27, 4877.

[88] a) N. E. Motl, J. F. Bondi, R. E. Schaak, Chem. Mater. 2012, 24, 1552; b) S. U. Lee, J. W. Hong, S. I. Choi, S. W. Han, J. Am. Chem. Soc. 2014, 136, 5221; c) X. G. Ding, Y. Zou, J. Jiang, J. Mater. Chem. 2012, 22, 23169.

[89] a) M. H. Liu, H. C. Zeng, Langmuir 2014, 30, 9838; b) Y. Feng, H. Liu, P. F. Wang, F. Ye, Q. Q. Tan, J. Yang, Sci. Rep-Uk. 2014, 4, 6204.

[90] a) S. Naya, T. Kume, R. Akashi, M. Fujishima, H. Tada, J. Am. Chem. Soc. 2018, 140, 1251; b) J. H. Yang, Y. Z. Guo, W. Z. Lu, R. B. Jiang, J. F. Wang, Adv. Mater. 2018, 30, 1802227.

[91] C. S. Shan, E. T. Martin, D. G. Peters, J. M. Zaleski, Chem. Mater. 2017, 29, 6030.

[92] a) L. Rodriguez-Lorenzo, R. de la Rica, R. A. Alvarez-Puebla, L. M. Liz-Marzan, M. M. Stevens, Nat. Mater. 2012, 11, 604; b) J. Langer, S. M. Novikov, L. M. Liz-Marzan, Nanotechnology 2015, 26, 322001 .

[93] A. Shiohara, S. M. Novikov, D. M. Solis, J. M. Taboada, F. Obelleiro, L. M. Liz-Marzan, J. Phys. Chem. C 2015, 119, 10836 .

[94] S. F. Shams, M. R. Ghazanfari, C. Schmitz-Antoniak, Nanomaterials-Basel 2019, 9, 97.

[95] J. R. McCarthy, R. Weissleder, Adv. Drug. Deliver. Rev. 2008, 60, 1241.

[96] X. Z. Zhu, H. K. Yip, X. L. Zhuo, R. B. Jiang, J. L. Chen, X. M. Zhu, Z. Yang, J. F. Wang, J. Am. Chem. Soc. 2017, 139, 13837.

[97] T. Chen, G. Chen, S. X. Xing, T. Wu, H. Y. Chen, Chem. Mater. 2010, 22, 3826.

[98] F. J. Zheng, W. Ke, L. X. Shi, H. Liu, Y. Zhao, Anal. Chem. 2019, 91, 11812.

[99] M. Y. Wang, J. Ioccozia, L. Sun, C. J. Lin, Z. Q. Lin, Energ. Environ. Sci. 2014, 7, 2182.

[100] G. Y. Yu, J. Qian, P. Zhang, B. Zhang, W. X. Zhang, W. F. Yan, G. Liu, Nat. Commun. $2019,10$.

[101] M. L. Brongersma, N. J. Halas, P. Nordlander, Nat. Nanotechnol. 2015, 10, 25.

[102] S. Rodal-Cedeira, V. Montes-Garcia, L. Polavarapu, D. M. Solis, H. Heidari, A. La Porta, M. Angiola, A. Martucci, J. M. Taboada, F. Obelleiro, S. Bals, J. Perez-Juste, I. Pastoriza-Santos, Chem. Mater. 2016, 28, 9169.

[103] B. H. Wu, D. Y. Liu, S. Mubeen, T. T. Chuong, M. Moskovits, G. D. Stucky, J. Am. Chem. Soc. 2016, 138, 1114.

[104] H. L. Jia, A. X. Du, H. Zhang, J. H. Yang, R. B. Jiang, J. F. Wang, C. Y. Zhang, J. Am. Chem. Soc. 2019, 141, 5083.

[105] Q. P. Lu, A. L. Wang, Y. Gong, W. Hao, H. F. Cheng, J. Z. Chen, B. Li, N. L. Yang, W. X. Niu, J. Wang, Y. F. Yu, X. Zhang, Y. Chen, Z. X. Fan, X. J. Wu, J. P. Chen, J. Luo, S. Z. Li, L. Gu, H. Zhang, Nat. Chem. 2018, 10, 456.

[106] a) L. H. V. Wang, S. Hu, Science 2012, 335, 1458; b) J. Xia, J. J. Yao, L. V. Wang, Prog. Electromagn. Res. 2014, 147, 1.

[107] J. K. Norskov, T. Bligaard, J. Rossmeisl, C. H. Christensen, Nat. Chem. 2009, 1, 37.

[108] X. Zhang, Z. S. Wu, X. Zhang, L. W. Li, Y. Y. Li, H. M. Xu, X. X. Li, X. L. Yu, Z. S. Zhang, Y. Y. Liang, H. L. Wang, Nat. Commun. 2017, 8, 14675.

[109] Z. C. Zhang, G. G. Liu, X. Y. Cui, B. Chen, Y. H. Zhu, Y. Gong, F. Saleem, S. B. Xi, Y. H. Du, A. Borgna, Z. C. Lai, Q. H. Zhang, B. Li, Y. Zong, Y. Han, L. Gu, H. Zhang, Adv. Mater. 2018, 30 . 


\section{WILEY-VCH}

[110] Q. P. Lu, A. L. Wang, H. F. Cheng, Y. Gong, Q. B. Yun, N. L. Yang, B. Li, B. Chen, Q. H. Zhang, Y. Zong, L. Gu, H. Zhang, Small 2018, 14.

[111] S. G. Kwon, G. Krylova, P. J. Phillips, R. F. Klie, S. Chattopadhyay, T. Shibata, E. E. Bunel, Y. Z. Liu, V. B. Prakapenka, B. Lee, E. V. Shevchenko, Nat. Mater. 2015, 14, 215. 